\title{
Teilnahme an mathematischen Vorkursen und langfristiger Studienerfolg. Eine empirische Untersuchung
}

\author{
Arne Gerdes (D) - Stefan Halverscheid • Susanne Schneider
}

Eingegangen: 31. Mai 2020 / Angenommen: 30. September 2021 / Online publiziert: 4. November 2021

(C) Der/die Autor(en) 2021

Zusammenfassung Mathematische Vorkurse stellen eine in Deutschland verbreitete Maßnahme zur fachlichen Unterstützung des Übergangs von Schule zu Studium dar. Der Beitrag geht der Frage der Wirksamkeit von Vorkursteilnahme, binär operationalisiert, hinsichtlich der langfristigen Kriterien des erfolgreichen Studienabschlusses nach acht Semestern und der Abschlussnote nach. Da es sich bei Vorkursangeboten in der Regel um optionale Teilnahmen handelt, wird zusätzlich die Selbstselektion der Studienanfängerinnen und -anfänger untersucht. Die Stichprobe bilden $N=2953$ Studierendenfachfälle verschiedener Bachelorstudiengänge der GeorgAugust-Universität Göttingen. Im Rahmen der Auswertungen zu Wirksamkeit und Selektion erfolgen die Modellierungen mit bayesscher linearer bzw. logistischer multipler Mehrebenenregression. Die Auswertung zur Wirksamkeit erfolgt auch mit zusätzlicher Entropie-Balancierung. Es zeigen sich unter Kontrolle weiterer Variablen positive Zusammenhänge sowohl von Vorkursteilnahme und erfolgreichem Studienabschluss als auch von Vorkursteilnahme und besseren Abschlussnoten. Eine Selektion der Teilnehmenden zeigt sich hinsichtlich des Geschlechts sowie der Note und des Bundeslands der Hochschulzugangsberechtigung.

Arne Gerdes $(\bowtie) \cdot$ Susanne Schneider

Didaktik der Physik, Fakultät für Physik, Georg-August-Universität Göttingen, Göttingen,

Deutschland

E-Mail: agerdes@uni-goettingen.de

Susanne Schneider

E-Mail: sschnei@uni-goettingen.de

Arne Gerdes

Fakultät für Mathematik und Informatik, Georg-August-Universität Göttingen, Göttingen,

Deutschland

Stefan Halverscheid

Mathematisches Institut, Fakultät für Mathematik und Informatik, Georg-August-Universität

Göttingen, Göttingen, Deutschland

E-Mail: stefan.halverscheid@mathematik.uni-goettingen.de 
Schlüsselwörter Vorkurs · Brückenkurs · Mathematik · Wirksamkeit · Studienerfolg $\cdot$ Studienabbruch

\title{
Pre-Courses in Mathematics and Long-Term Academic Success. An Empirical Study
}

\begin{abstract}
Pre-courses in mathematics are a common offer to support the transition from school to university in Germany. This paper examines the effectiveness of preparatory courses regarding the long-term criteria of successful completion of studies and final grades. As pre-courses are usually voluntary or optional, additional selection effects are examined. The sample ( $N=2953$ cases $)$ stems from different undergraduate programmes at Göttingen University. In the context of the evaluation of effectiveness and selection effects, the modelling is carried out with Bayesian linear and logistic multiple multilevel regression. The evaluation of effectiveness is also done with additional entropy balancing. The results show, among other things, positive correlations between participation in the pre-course and successful completion of the degree programme as well as better final grades. Selection effects can be seen for gender as well as the final grade and the state of the previously attended school.
\end{abstract}

Keywords Pre-course $\cdot$ Math $\cdot$ Effectiveness $\cdot$ Success $\cdot$ Dropout

\section{Einleitung}

Die Förderung von Studienerfolg und damit verbunden die Verringerung von Studienabbruchquoten stellen hochschulpolitisch wichtige Ziele dar, die u. a. über den Fachkräftebedarf motiviert werden (BMBF 2019). Fokussiert wird dabei häufig auf die Übergänge in das Hochschulsystem und die Studieneingangsphase (Schaeper 2020; Moser-Fendel und Wessel 2019; Blömeke 2016; Rach 2014). So erfolgen rund die Hälfte der Studienabbrüche in Bachelorstudiengängen an deutschen Hochschulen im ersten Studienjahr (Heublein et al. 2017). Hieran ansetzend werden seit langem Maßnahmen entwickelt und implementiert, die Studienerfolg und -abbruch adressieren. Als eine bundesweite Initiative sei das Bund-Länder-Programm Qualitätspakt Lehre (QPL) genannt. Einen exemplarischen Einblick in die Vielfalt an Maßnahmen am Übergang von Schule zu Studium zur Verbesserung des Studienerfolgs geben Key und Hill (2018) und speziell in mathematikdidaktischer Hinsicht Roth et al. (2015) sowie Hoppenbrock et al. (2016).

Studienabbrüche werden dabei als multikausal angesehen. Am häufigsten werden von Studienabbrecherinnen und -abbrechern Leistungsaspekte wie mangelnde fachliche Voraussetzungen als führender Grund des Abbruchs angegeben, insbesondere auch in Studiengängen mit mathematischen Studieninhalten (Heublein et al. 2017; Albrecht und Nordmeier 2011). Konsistent zeigen Schulnoten und Vorwissen einen Zusammenhang zu verschiedenen Operationalisierungen des Studienerfolgs (Trapmann et al. 2007; Albrecht und Nordmeier 2011; Larsen et al. 2013; Halverscheid und Pustelnik 2013; Freyer et al. 2014; Buschhüter et al. 2016; Rach und Heinze 
2017; Müller et al. 2018; Klein et al. 2018; Fleischer et al. 2019; Binder et al. 2019; Rach und Ufer 2020; Burkholder et al. 2021). In einer Typologie von Exmatrikulierten ohne Abschluss stellten in den Naturwissenschaften überforderte Studierende die größte Gruppe (Blüthmann et al. 2012). Zu den führenden Schwierigkeiten explizit zu Studienbeginn gehören die Leistungsanforderungen (Bargel 2015). Unzureichende fachliche Voraussetzungen stellen dementsprechend bedeutsame Faktoren des Studienabbruchs dar (Heublein et al. 2010, 2017). Heublein et al. (2010) ergänzen, dass mit zunehmender Zeit zwischen Erwerb der Hochschulzugangsberechtigung (HZB) und Studienbeginn Studierende häufiger berichten, dass studienrelevantes Vorwissen in Vergessenheit geraten ist. Die Fokussierung dieses Beitrags auf Vorkurse und ihre Wirksamkeit hinsichtlich des erfolgreichen Studienabschlusses soll nicht darüber hinwegtäuschen, dass es sich beim Prozess des Studiums und des Studienabbruchs um ein komplexes, multikausales Gefüge handelt. Einen Überblick zu Studienabbruch, Ursachen und Folgen geben Neugebauer et al. (2019). Larsen et al. (2013) bzw. zusammenfassend Kehm et al. (2019) fanden in einer internationalen, systematischen Reviewstudie zu Studienabbruch bei hoher Zahl an Untersuchungsmerkmalen insbesondere Evidenz in den Bereichen des sozio-demografischen Hintergrunds und persönlicher Merkmale, der institutionellen Bedingungen und der akademischen Integration. Hier gehen männliches Geschlecht und eine zeitliche Lücke mit höherer und gute Schulnoten und gute Klausurergebnisse mit niedrigerer Wahrscheinlichkeit des Studienabbruchs einher.

Der Befundlage zu Vorwissen und Überforderung entsprechend wird nach Maßnahmen gesucht, die Schwierigkeiten fachlicher Voraussetzungen adressieren. In einer qualitativen Auswertung der QPL-Maßnahmen leiteten Bosse et al. (2019) eine Typisierung von neun Maßnahmen-Kategorien in der Studieneingangsphase ab, exemplarische Vertreter zur Vermittlung von Fachwissen sind dabei mathematische Vorkurse. Neugebauer et al. (2019) stellen zu diesen Maßnahmen fest: „Allerdings gibt es bislang kaum belastbare Erkenntnisse zu deren Wirksamkeit, da Evaluationen nur selten dem Problem der Selbstselektion durch randomisierte Kontrollstudien oder quasi-experimentelle Untersuchungen begegnen.“ (Neugebauer et al. 2019, S. 1030)

Im vorliegenden Beitrag soll der Frage nachgegangen werden, ob in universitären Studiengängen mit mathematischen Studieninhalten die Teilnahme, binär operationalisiert als Teilnahme ja/nein, an solchen mathematischen Vorkursangeboten am Übergang von Schule zu Studium einen positiven Zusammenhang mit erfolgreichem Studienabschluss und -note zeigt. Näher betrachtet werden Fragen zu Interaktionseffekten mit einzelnen individuellen Variablen der Studierenden. Differenzierenden Fragen zu Oberflächen- und Tiefenmerkmalen der Vorkurse wird aufgrund der Datenbasis empirisch nicht nachgegangen. Die Frage nach dem erfolgreichen Studienabschluss wählt dabei explizit einen auf die langfristige Wirksamkeit der Angebote abzielenden Kriterienbereich. Der Frage warum bzw. wie Vorkurse eine entsprechende Wirksamkeit erzielen, kann im vorliegenden Studiendesign nicht nachgegangen werden. Da es sich bei Vorkursen in der Regel um optionale Angebote handelt, soll zusätzlich eine Selbstselektion von Teilnehmenden untersucht werden. 


\section{Mathematische Vorkurse}

Mathematische Vorkurse werden u. a. über hohe Studienabbruchquoten und niedrige Bestehensquoten in Klausuren motiviert (z. B. Reichersdorfer et al. 2014; Kürten 2020). Sie werden als Antwort auf mangelndes Vorwissen verstanden (z. B. Abel und Weber 2014) bzw. auf eine Heterogenität der Studienanfängerinnen und -anfänger (z. B. Greefrath et al. 2015; Halverscheid et al. 2014).

Mathematische Vorkurse, auch mit uneinheitlichen Abweichungen Vorbereitungskurse, Propädeutika, Brückenkurse u. ä. genannt, werden seit langer Zeit am Übergang Schule-Studium angeboten. So gab es zum Beispiel an der in dieser Untersuchung betrachteten Universität Göttingen bereits 1969 einen der Vorlesungszeit vorgelagerten, einmonatigen mathematischen Vorbereitungskurs als Blockveranstaltung (Georg-August-Universität Göttingen 1969). Im Rahmen des bundesweiten Studieneingangstests von Physikstudierenden 1978 beschrieben Krause und Reiners-Logothetidou (1981) eine bundesweite Vielfalt an Vorkursangeboten, mit regional/bundesland-spezifischen Angebotsunterschieden. Eine erneute Durchführung der Studie 2013 zeigt, dass sich der Anteil von Vorkursteilnehmenden unter Physikstudierenden in Deutschland dabei deutlich von $27 \%$ im Jahr 1978 auf $80 \%$ im Jahr 2013 erhöht hat (Krause und Reiners-Logothetidou 1981; Buschhüter et al. 2016). Eine Umfrage unter den Mitglieder-Hochschulen der Konferenz der mathematischen Fachbereiche 2018 zeigte, dass inzwischen fast alle von diesen mathematische Vorkurse anbieten, wobei rund die Hälfte der Kurse für Nicht-Mathematikstudierende angeboten wird (Kallweit et al. 2018). Die Dauer der Kurse liegt zwischen ein bis sechs Wochen, mit einem Median von zwei Wochen (Kallweit et al. 2018). Es liegen mit cosh-Katalog für WiMINT-Studiengänge (cosh 2014), MaLeMINT (Neumann et al. 2017) und MWK (2019) für MINT-Studiengänge und KFP (2012) für PhysikStudiengänge mehrere aktuelle Rahmen vor, die inhaltliche Orientierung zur curricularen Gestaltung von mathematischen Vorkursen geben. Es wurden zahlreiche Lehrbücher zu Vorkursen veröffentlicht, die inhaltliche Realisierungen dokumentieren (z.B. Hefft 2018; Cramer und Nešlehová 2018; Purkert 2014). Eine Übersicht von vielseitig exemplarischen Vorkurskonzeptionen geben Bausch et al. (2014).

Das Angebot mathematischer Vorkurse ist Teil der gemeinsamen Handlungsempfehlung von DMV, GDM und MNU zum Übergang Schule - Hochschule, die auch die Forschungsbegleitung und Evaluation der Maßnahmen als Rahmenbedingung vorsieht (DMV et al. 2019). Diesem Anspruch entsprechend soll die vorliegende Untersuchung zur Wirksamkeit von Vorkursen einen Beitrag hinsichtlich der Betrachtung langfristiger Kriterien des Studienerfolgs leisten.

Wie geschildert wird Vorwissen als eine der zentralen Variablen für Studienabbruch insbesondere in mathematischen Studiengängen gefunden, Vorkurse werden als das Vorwissen fördernde Maßnahme implementiert. Da wie von Rach und Ufer (2020) ausgeführt, Vorwissen zentrale Bedeutung für die Studieneingangsphase in konstruktivistischer Perspektive durch die Konstruktion neuen Wissens auf Grundlage bestehenden Wissens zukommt, lässt sich dieser Gedanke wiederum auch auf sich anschließende, auf vorherigen Veranstaltungen aufbauende, Module fortsetzen (wie von z.B. einer Physik-I- zu einer Physik-II-Vorlesung wie bei Burkholder et al. 2021). Eine aggregierte, langfristige Wirkung von Vorkursen lässt sich hierbei dann 
in erfolgreichem Studienabschluss und Abschlussnote als Aggregat der studienbegleitenden Modulnoten prüfen.

\subsection{Wirksamkeit von mathematischen Vorkursen}

Bei allen Maßnahmen stellen sich Fragen zu Wirkungen und Wirksamkeiten. In einer systematischen Reviewstudie von Publikationen zum Studienabbruch in Europa für den Zeitraum von 2000 bis 2012 fanden Larsen et al. (2013) insgesamt 62 einschlägige Publikationen den Studienabbruch betreffend, darunter nur 11, die sich Interventionen widmeten. Unter diesen fanden sie nur drei Publikationen, die den Qualitätskriterien des Reviews entsprachen, darunter keine Publikation, die die Wirksamkeit von Vorkursen hinsichtlich des Studienabbruchs untersuchte (Larsen et al. 2013). Auch bei elaborierten Designs der Aktionsforschung zu Vorkursen wie bei Kürten (2020) bleiben, wie die Autorin explizit anmerkt, übergeordnete Ziele wie die Reduktion des Studienabbruchs unerfasst. Dies scheint allgemein eine Herausforderung für die Weiterentwicklung der Angebote darzustellen. In jüngster Zeit kam Tieben (2019) weiterhin zu der Feststellung, dass, bis zu Erscheinen ihrer Arbeit, für den deutschsprachigen Raum keine Publikationen bzgl. des Zusammenhangs von Vorkursbesuch und Studienabbruch vorliegen. Für Studierende in Ingenieurstudiengängen fand Tieben (2019) dabei in einer elaborierten Auswertung unter Einbezug aller ihr vorliegender Kontrollvariablen weder bei Universitäten noch Fachhochschulen einen auf 5\%-Niveau signifikanten Zusammenhang von Vorkursteilnahme und Studienabbruch. Die Autorin diskutiert dabei, dass einige aufgenommene Kontrollvariablen zeitlich nach dem ggf. erfolgten Vorkursbesuch gemessen wurden und durch diesen hätten beeinflusst werden können, was die Rezeptionsmöglichkeit hinsichtlich der Wirkung der Vorkursteilnahme einschränkt. Ohne Einbezug von Kontrollvariablen wird von Tieben (2019) für Studierende an Universitäten ein auf $5 \%$-Niveau signifikanter Zusammenhang von Vorkursteilnahme und Studienabbruch gefunden, wobei Vorkursteilnehmende seltener ihr Studium abbrechen als Nichtteilnehmende.

Betrachtet man statt des erfolgreichen Studienabschlusses vorgelagerte kurz- bzw. mittelfristige Kriterien, so sind die Befunde unterschiedlich. Voßkamp und Laging (2014) fanden für Studierende der Wirtschaftswissenschaften einer Universität unter Kontrolle weiterer Variablen einen positiven Effekt der Vorkursteilnahme auf das Ergebnis eines Mathematiktests zu Studienbeginn. Für die Klausurergebnisse von mathematischen Anfangsveranstaltungen (Lineare Algebra, Analysis) von Informatik- und Elektroingenieurstudierenden an einer Universität fanden Greefrath et al. (2017) unter Kontrolle weiterer Variablen keinen Zusammenhang zur Vorkursteilnahme. Hier schränkt die Aufnahme des Vorwissens, gemessen zeitlich nach der ggf. erfolgten Vorkursteilnahme, als Kontrollvariable die Rezeptionsmöglichkeit der Befunde ein. Ohne Einbezug von Kontrollvariablen fanden sie in vier Einzeltests, nach den zwei Studiengängen und den zwei Veranstaltungen getrennt, im Falle der Informatikstudierenden in den Klausurergebnissen in Analysis einen Unterschied zugunsten der Vorkursteilnehmenden, während in den anderen drei Konstellationen keine Unterschiede gefunden wurden. Kürten (2020) fand für Studierende der Ingenieurwissenschaften einer Fachhochschule positive Zusammenhänge von Vor- 
kursteilnahme und Klausurerfolgen. Heublein et al. (2017) berichten, dass in einer Prüfung keine Zusammenhänge von Teilnahme an mathematischen Vorkursen und u.a. selbsteingeschätzter, fachlicher Bewältigung des Studieneinstiegs festgestellt werden konnte, wobei keine Kontrollvariablen berücksichtigt wurden. Büchele (2020) fand für Studierende der Wirtschaftswissenschaften einer Universität für semesterbegleitende Brückenkurse positive Zusammenhänge u.a. zum Klausurerfolg in Mathematik.

Jenseits entsprechender fachwissenschaftlicher Kompetenzunterschiede können auch andere Wirkungen und deren zeitliche Dauer untersucht werden. Rach et al. (2014) zeigten, dass die von ihnen untersuchten Vorkursteilnehmenden gegenüber Nicht-Teilnehmenden zu Beginn der gemeinsamen Vorlesungszeit adäquatere Erwartungen hinsichtlich der fachwissenschaftlichen Studienanforderungen ihres (Wirtschafts-)Mathematikstudiums aufwiesen. Eine Messung vier Wochen später zeigte, dass sich die Erwartungen anglichen und die Unterschiede zwischen Vorkursteilnehmenden und Nicht-Teilnehmenden hinsichtlich dessen somit nur kurzfristig blieben. Hinsichtlich der Selbstwirksamkeitserwartungen dokumentieren Kürten (2020) in einer Pre-Post-Erhebung und Fischer (2014) in retrospektiver Befragung Zuwächse von Selbstwirksamkeitserwartungen während der Vorkurse.

Die Nützlichkeit der Vorkurse wird in der Zielgruppe selbst uneindeutig beurteilt. Sie werden in der Untersuchung von Heublein et al. (2017) von $40 \%$ der Absolventinnen und Absolventen als auch von 55\% der Studienabbrecherinnen und -abbrecher als nützlich eingeschätzt, bei Bargel (2015) von $59 \%$ der Teilnehmenden.

Den Gedanken der Studienvorbereitung aufgreifend, sind im US-amerikanischen Raum remedial-math courses verbreitet. Anders als die in diesem Artikel fokussierten Vorkurse im deutschen Bildungssystem, als freiwillige, relativ kurze Blockveranstaltungen vor Studienbeginn, handelt es sich hier in der Regel um verpflichtende Semesterkurse, die anhand von variierenden Kriterien wie Einstufungstests zu belegen sind (Valentine et al. 2017). Die Kurse werden dabei auch, u. a. hinsichtlich ihrer Kosten, ihres zusätzlichen Zeitbedarfs und der Messgüte herangezogener Einstufungskriterien, kritisch diskutiert (Valentine et al. 2017). Die kriterienbasierte Zuordnung zur verpflichtenden Maßnahmenwahrnehmung macht es herausfordernd, Effekte des Förderungsunterrichts in Hinblick auf den Studienerfolg adäquat zu bestimmen (z. B. Valentine et al. 2017; Moss et al. 2014). Moss et al. (2014) nahmen dies zum Anlass, mit einer Kombination von randomisiertem Experiment, hier der randomisierten Zuordnung zu Maßnahme bzw. Kontrollgruppe innerhalb eines Intervalls um den Kriteriumswert, und Regressions-Diskontinuitäts-Analyse (RDAnalyse) die Wirksamkeit mathematischer remedial courses zu untersuchen. Sie fanden eine positive Wirksamkeit hinsichtlich der Studiennoten. Eine Metaanalyse von RD-Analysen remedial courses mathematischer und sprachlicher Ausrichtung von Valentine et al. (2017) kommt zu dem Ergebnis, dass die Zuweisung negative Einflüsse in Hinblick auf erworbene Credits, erfolgreiches Absolvieren des korrespondierenden Zielkurses und erfolgreichen Studienabschluss hat. Für Studiennoten wurde kein signifikanter Unterschied gefunden. Der Befund zu erworbenen Credits konnte nach Kursfach differenziert ausgewertet werden: während bei sprachlichen Kursen ein negativer Effekt gefunden wurde, wurde bei mathematischen Kursen kein von null verschiedener Effekt signifikant. Für kriterienbasiert zugeordnete, kombi- 
nierte mathematisch-sprachliche Förderkurse in Italien fanden De Paola und Scoppa (2014) mit einer RD-Analyse einen positiven Effekt der Kursteilnahme auf die Zahl erworbener Credits und die Verringerung von Studienabbruch, jeweils in einem Beobachtungszeitraum von zwei Jahren. Ohne dies prüfen zu können, vermuten sie aufgrund eines als stärker eingeschätzten Effekts auf Abbruch als auf erworbene Credits, dass entsprechende Förderkurse möglicherweise insbesondere für leistungsschwächere Studierende hilfreich sein könnten. Der Frage, ob Kurse je nach anfänglichem Leistungsstand unterschiedliche Effekte hinsichtlich verschiedener mittelbis langfristiger Ziele zeigen, gingen Boatman und Long (2018) mit RD-Analyse nach, indem nicht ein, sondern zwei Diskontinuitäten betrachtet wurden. Insgesamt wurden auf Mathematikförderkurse bezogen überwiegend negative Effekte bis von null nicht zu unterscheidende Effekte gefunden, mit der Beobachtung, dass in der Tendenz signifikant negative Effekte eher an der leistungshöheren Diskontinuität gefunden wurden.

\subsection{Selektion: Wer nimmt an Vorkursen teil?}

Bargel (2015) dokumentiert eine Nutzungsquote unter den Studienanfängerinnen und -anfängern von $52 \%$ von Brückenkursen, wo diese angeboten werden bzw. insgesamt $44 \%$ in Wirtschaftswissenschaften, $45 \%$ in Naturwissenschaften/ Mathematik und $48 \%$ in Ingenieurwissenschaften. Tieben (2019) berichtet von einer Teilnahmequote von insgesamt $55 \%$ in Ingenieurwissenschaften, speziell an Fachhochschulen von $44 \%$ und an Universitäten von $64 \%$. Der optionale Charakter von Vorkursangeboten, sowohl seitens der Hochschule in deren Angebot als auch seitens der Studierenden in der ggf. Wahrnehmung des Angebots, drückt sich in diesen Zahlen aus.

Damit ist die Teilnahme Ergebnis eines Entscheidungsprozesses, über dessen Details bislang kaum etwas bekannt ist. Tieben (2019) schildert, dass die Teilnahmeentscheidung sowohl Kenntnis der Existenz des Angebots, Motivation zur Teilnahme und wahrgenommene Notwendigkeit des Besuchs bedürfe. Bezogen auf Studienanfängerinnen und -anfänger von ingenieurwissenschaftlichen Studiengängen sei davon auszugehen, dass mathematische Kompetenzen von diesen als bedeutsam für ihr Studium erkannt würden. Als Kriterien, die eine Entscheidung zur Vorkursteilnahme beeinflussen könnten, werden somit u. a. schulische Noten, insbesondere diejenigen in Mathematik, und entsprechende Selbsteinschätzungen diskutiert, wobei diese jeweils nur eingeschränkt zur Abschätzung der eigenen Kompetenzen bzw. bestehender Defizite geeignet seien. Auch könne die individuelle Leistungsorientierung Auswirkung auf die Teilnahmeentscheidung haben. Bezogen auf universitäre Studiengänge, auf die wir uns vorliegend fokussieren, der Ingenieurwissenschaften zeigt in der Untersuchung von Tieben (2019) empirisch keine der angesprochenen Variablen (Note der HZB, Mathematiknote, Akademisches Selbstkonzept und Selbsteinschätzung der Studienvorbereitung allgemein und auf Mathematik bezogen, Leistungsorientierung) auf einem 5\%-Niveau einen signifikanten Unterschied zwischen den Gruppen der Teilnehmenden und Nicht-Teilnehmenden. Im Rahmen von 15 Einzeltests wurden von Tieben (2019) weitere Variablen geprüft; hier zeigen, weiterhin bezogen auf die universitären Studiengänge, u. a. auch demografische Va- 
riablen wie Geschlecht und Alter zu Studienbeginn keinen signifikanten Unterschied auf $5 \%$-Niveau, einzig der Berufsstatus der Eltern (ISEI) zeigt einen signifikanten Unterschied von Teilnehmenden und Nicht-Teilnehmenden, mit höherer Ausprägung des Berufsstatus bei den Nicht-Teilnehmenden. Eine multiple Regression mit Vorkursbesuch als abhängiger Variablen wird neben den Einzeltests nicht berichtet.

Wir ergänzen, dass neben den angesprochenen Aspekten auch die Möglichkeit der Teilnahme an zeitlich vorgelagerten Blockveranstaltungen bestehen muss bzw. Hürden denkbar sind, wie geografische Einzugsgebiete und Überschneidungen mit anderen Verpflichtungen, wie Vereinbarkeit von Studium, Beruf und Familie.

Büchele (2020) berichtet für die Teilnahme von Studierenden der Wirtschaftswissenschaften für semesterbegleitende, freiwillige Brückenkurse als Ergebnis einer multiplen logistischen Regression, dass die Wahrscheinlichkeit der Teilnahme mit höherer selbsteingeschätzter Mathematikfähigkeit steigt, während Geschlecht, Kohorte, vorheriger Besuch des Moduls, Jahrgangstufe der HZB, HZB- und Mathematiknote, vergangene Zeit seit Erwerb der HZB und der vorherige Besuch eines Vorkurses keine signifikanten Effekte zeigen.

Heublein et al. (2010) berichten, dass Studierende mit sehr guten HZB-Noten häufiger Vorkurse besuchten als solche mit schlechteren Noten. Dies wird mit der Einschätzung verbunden, dass damit gerade diejenigen Studierenden, die entsprechende Unterstützung am ehesten benötigen, die Angebote seltener wahrnehmen. Hierin wird eine ungenügende Steuerung gesehen.

Von Bargel (2015) berichtete Zusammenhänge u.a. von Alter und Geschlecht in Hinblick auf den Besuch von Vorkursen lassen sich nur eingeschränkt interpretieren, da alle Fachrichtungen der Hochschulen gemeinsam betrachtet wurden, u.a. die Existenz von Vorkursangeboten je nach Fachrichtung aber als unterschiedlich angenommen werden muss. Einige Arbeiten im Forschungsbereich sind in Hinblick auf die Frage von Selektion der Teilnehmenden aufgrund fehlender statistischer Prüfung, nur eingeschränkt passender statistischer Prüfung oder fehlender statistischer Angaben für die vorliegende Arbeit in ihrer Rezeption eingeschränkt.

Während im Bereich der Untersuchung zur Wirksamkeit von Förderangeboten am Übergang mit Einschränkungen der Vergleichbarkeit von Bildungssystemen und Angeboten auf US-amerikanische, verpflichtend zugeordnete Förderkurse Bezug genommen werden konnte, ist dieses im Fall der vorliegenden Betrachtung von Selbstselektion zu den hier betrachteten freiwilligen Kursen entsprechend nicht möglich.

Dieses hat Auswirkungen auf die methodischen Herangehensweisen zur Reduzierung möglicher Verzerrungen durch Selektion bei der Untersuchung der Wirksamkeit der Angebote. So finden sich wie oben gesehen bei der Untersuchung von Kursen mit anhand eines Grenzwerts selektierten Teilnehmenden passend RD-Analysen, während bei der Untersuchung von optionalen Kursen mit Selbstselektion z. B. ein Matching wie bei Büchele (2020) oder eine Balancierung wie bei Tieben (2019) vorgenommen wird. 


\section{Forschungsfragen}

Es zeigt sich, dass bezüglich der Wirkungen und Wirksamkeiten von mathematischen Vorkursen noch ein Forschungsbedarf besteht. Dies gilt insbesondere, wenn nach der langfristigen Wirksamkeit bzgl. des Studienerfolgs in einer breiten Verteilung von Studiengängen gefragt wird.

Es werden optionale mathematische Vorkurse am Übergang in das Hochschulsystem in Hinblick auf Teilnahmeselektion und Wirksamkeit hinsichtlich des langfristigen Studienerfolgs der teilnehmenden Studierenden betrachtet.

Den optionalen Charakter der Angebotsnutzung adressiert eine erste Frage:

(1) Welche Selektion der Vorkursteilnehmenden existiert hinsichtlich der individuellen Variablen Geschlecht, Note und Bundesland der HZB sowie zeitlichem Abstand zwischen Erwerb der HZB und Studienbeginn?

Dies ist aus mehreren Perspektiven motiviert: Wird eine positive Wirksamkeit angenommen, so stellt sich aus Perspektive der Chancengleichheit die Aufgabe zu prüfen, ob es in Form von beobachteter Selektion (z. B. in Hinblick auf Geschlecht, Zeit seit HZB oder regionale Herkunft) Anhaltspunkte dafür gibt, die auf mögliche Diskriminierung bzw. Hürden bei der Angebotsannahme hindeuten könnten. Gefundene Selektion kann hier weitergehende Untersuchungen motivieren. Ebenfalls aus praktischer Perspektive erscheint es relevant zu betrachten, ob Studierende insbesondere hinsichtlich ihrer schulischen Leistung Unterschiede in der Angebotsannahme zeigen, da bei ungünstigem Zusammenhang besonders relevante Zielgruppen weniger erreicht würden. Für die statistische Auswertung zur Wirksamkeit ist daneben die Frage der möglichen Selektion von Teilnehmenden und Nicht-Teilnehmenden relevant, da hier ggf. statistische Korrekturen hilfreich sein können. Der Frage wird, der Motivation der Frage und der Forschungslage entsprechend, explorativ nachgegangen.

Der Wirksamkeit einer Vorkursteilnahme bzgl. des langfristigen Studienerfolgs wird in einer zweiten Forschungsfrage nachgegangen:

(2) Welchen Effekt hat die Vorkursteilnahme auf den langfristigen Studienerfolg der Teilnehmenden in Hinblick auf (a) den erfolgreichen Studienabschluss innerhalb von acht Semestern und (b) die Abschlussnote?

Entsprechend den Überlegungen zur Bedeutung des Vorwissens für den Studienerfolg und der Vorkurse als entsprechender Fördermaßnahmen prüfen wir die Vermutung eines positiven Effekts von Vorkursteilnahme auf Studienerfolg.

Für Studienerfolg kommen hier, wie der oben ausgeführten Literatur entnommen werden kann, verschiedene kurz-, mittel- und langfristige Kriterien in Betracht. Im vorliegenden Beitrag möchten wir uns den langfristigen Kriterien widmen und betrachten hier konkret den erfolgreichen Studienabschluss der Bachelorstudiengänge innerhalb von acht Semestern sowie die Abschlussnote.

Die Wahl des Betrachtungszeitraums von acht Semestern bei einer Regelstudienzeit von sechs Semestern folgt dabei in Anlehnung an den Indikator „Abschlüsse in angemessener Zeit" (CHE 2021) des CHE-Hochschulrankings, der bezogen auf Bachelorstudiengänge in der Regel Abschlüsse in Regelstudienzeit plus zwei Semestern 
betrachtet, und in Anlehnung an den vom Wissenschaftsrat u. a. zu Steuerungszwecken diskutierten Parameter der ,Zahl aller Studierenden in der Regelstudienzeit plus zwei Semester“ (Wissenschaftsrat 2018, S. 49) sowie forschungspraktischen Limitierungen des Datensatzes.

Als Variation von Forschungsfrage 2 fragen wir drittens:

(3) Werden die von Forschungsfrage 2 adressierten Effekte durch (a) die Zeitdifferenz zwischen Erwerb der HZB und Studienbeginn und (b) die HZB-Note moderiert?

Entsprechend des Befunds von Heublein et al. (2010), dass Studierende mit größerer Zeitdifferenz zwischen Erwerb der HZB und Studienbeginn häufiger davon berichten, dass studienrelevantes Vorwissen in Vergessenheit geraten ist und entsprechend einer Intention von Vorkursen, in Vergessenheit geratenes Vorwissen zu wiederholen, prüfen wir entsprechend die Vermutung, dass eine Vorkursteilnahme umso effektstärker ist, je länger der Erwerb der HZB zeitlich zurückliegt.

Entsprechend der aus praktischen Gesichtspunkten relevanten und in Anlehnung an den in der Literatur insbesondere im Kontext von RD-Analysen aufgeworfenen Aspekt der Wirksamkeit in Abhängigkeit des Vorwissens, prüfen wir, ob die Effektstärke einer Vorkursteilnahme mit der HZB-Note der Teilnehmenden variiert.

\section{Methode}

Die diesem Beitrag zugrunde liegenden Daten und Auswertungen sind Teil des Forschungsprojekts Göttingen Learning Analytics (GLA), welches sich der Forschung zu Studienwahl, -erfolg und -abbruch anhand von Fragebogenerhebungen, Testungen und Registerdaten der Georg-August-Universität Göttingen widmet. Das Projekt wurde vom Datenschutzbeauftragten der Universität Göttingen bzw. dessen Büro datenschutzrechtlich geprüft und genehmigt. Die Daten wurden pseudonym zusammengeführt, alle Auswertungen erfolgen anonym und aggregiert. Aus der Kombination von Matrikelnummer als erstem Pseudonym und einer weiteren Zeichenfolge (Salt) wurde dazu mit einer Hashfunktion ein zweites Pseudonym generiert. Mit diesem wurden die Daten pseudonym zusammengeführt. Entnommen wurde daraus eine anonyme und aggregierte Auswertung.

\subsection{Stichprobe}

Es werden $N=2953$ Studierendenfachfälle (39\% weiblich) der Georg-AugustUniversität Göttingen betrachtet, welche in ihrem ersten Hochschulsemester in den Wintersemestern 2012/2013 bzw. 2013/2014 am Semesterbeginn für einen von 19 betrachteten Bachelorstudiengängen mit mathematischen Studieninhalten immatrikuliert waren und ein Studium aktiv aufgenommen haben (siehe Tab. 1).

Das erste Hochschulsemester wird (statt ggf. des ersten Fachsemesters) betrachtet, um den Übergang in das Hochschulstudium zu modellieren. Die Stichprobe wird mit der Bedingung der aktiven Studienaufnahme gebildet, um einer möglichen Verzerrung der Wirkungsabschätzung zugunsten der Vorkurse zu begegnen. 
Tab. 1 Stichprobe: Studierendenfachfälle nach Studiengang und Abschlussziel

\begin{tabular}{|c|c|c|c|}
\hline & B.Sc. B.A. & B.A.-2fach & B.A.-LA \\
\hline \multicolumn{4}{|c|}{ Fakultät für Agrarwissenschaften } \\
\hline Agrarwissenschaften & 509 & & \\
\hline \multicolumn{4}{|c|}{ Fakultät für Biologie und Psychologie } \\
\hline Biologie & 291 & & 60 \\
\hline $\begin{array}{l}\text { Biologische Diversität } \\
\text { und Ökologie }\end{array}$ & 72 & & \\
\hline \multicolumn{4}{|c|}{ Fakultät für Forstwissenschaften und Waldökologie } \\
\hline $\begin{array}{l}\text { Forstwissenschaften und } \\
\text { Waldökologie }\end{array}$ & 288 & & \\
\hline \multicolumn{4}{|c|}{ Fakultät für Geowissenschaften und Geographie } \\
\hline Geowissenschaften & 78 & & \\
\hline \multicolumn{4}{|c|}{ Fakultät für Mathematik und Informatik } \\
\hline Angewandte Informatik & 94 & & \\
\hline Informatik & & & 18 \\
\hline Mathematik & 105 & 3 & 122 \\
\hline \multicolumn{4}{|l|}{ Fakultät für Physik } \\
\hline Physik & 309 & 3 & 29 \\
\hline \multicolumn{4}{|c|}{ Wirtschaftswissenschaftliche Fakultät } \\
\hline Betriebswirtschaftslehre & 564 & & \\
\hline Volkswirtschaftslehre & 206 & 185 & \\
\hline Wirtschaftsinformatik & 95 & & \\
\hline Wirtschaftspädagogik & 107 & & \\
\hline
\end{tabular}

Die Bedingung wird operationalisiert als die Anmeldung zu mindestens einer Prüfungsvorleistung oder Prüfungsleistung im ersten Semester. Es werden so 174 Fälle nicht in die Stichprobe aufgenommen. Weitere 11 Fälle werden nicht aufgenommen, da ihrem Datensatz eine Note der HZB fehlt.

\subsection{Datenbezug und Operationalisierung}

Den Registerdaten der Universität werden Geschlecht, HZB-Note, Studiengang inklusive Abschlussziel, Status des Abschlusses im achten Semester nach Studienbeginn (binär) und, sofern ein Abschluss vorliegt, Abschlussnote entnommen. Der zeitliche Abstand zwischen Erwerb der HZB und Studienbeginn wird aus den jeweiligen Datumsangaben als Differenz in Tagen berechnet. Aus dem Landkreis der HZB wird abgeleitet, ob die HZB in Niedersachsen (Nds.), dem Bundesland der betrachteten Universität, erworben wurde.

Der langfristige Studienerfolg wird vorliegend entsprechend in zweierlei Hinsicht als (a) erfolgreiche Absolvierung des gewählten Studiengangs innerhalb von acht Semestern seit Studienaufnahme und (b) über ggf. die Abschlussnote operationalisiert. Vorkursteilnahme wird binär über die Teilnahme an einem einführenden Mathematik-Test im Rahmen des Vorkursangebots operationalisiert.

Die einzelnen Kurse bestehen aus Vorlesungen und tutoriell angeleiteten Übungen in Kleingruppen und haben einen Umfang von ein bis drei Wochen äquivalent zu ein 
bis drei SWS. Bei allen Vorkursen handelt es sich um Präsenzveranstaltungen. Der Besuch aller betrachteten Kurse ist optional. Die Curricula der Kurse sind jeweils auf die adressierten Studierenden der verschiedenen Bachelorstudiengänge abgestimmt. Rolf et al. (2018) schlagen zur Strukturierung der Ziele von Vorkursen vier Zielbereiche vor, die sich auf Wissen, Handlung, Einstellungen und das universitäre System beziehen. Von den darin von Rolf et al. (2018) jeweils zusammengestellten Zielkategorien sprechen alle hier eingehenden Kurse im Bereich des Wissens schulische Mathematik und mathematische Fachsprache als Hauptziele an. Das Vorkursangebot für Studierende der Mathematik, Informatik und Physik hat darüber hinaus Ziele in der universitären Mathematik. Im Bereich der Handlung sprechen alle Kurse als Ziel mathematische Arbeitsweisen und in Hinblick auf den Bereich des universitären Systems soziale Kontakte als Ziel an. Letzteres ist eng mit dem Präsenzformat verbunden. Im Bereich der Einstellungen liegen keine Hauptziele der Kurse. Letzteres deckt sich mit dem Befund zu den exemplarischen Vorkursangeboten in Rolf et al. (2018). Eine exemplarische Übersicht zu Themen und Ablauf des an Teilnehmenden stärksten eingehenden Vorkursangebots für Studierende der Wirtschaftswissenschaften gibt Schnoor (2012).

\subsection{Statistische Auswertung}

Die Modellierung der Vorkursteilnahme erfolgt mit einer bayesschen logistischen multiplen Mehrebenenregression. Die Modellierung des Studienabschlusses bzw. der Abschlussnote erfolgt mit einer bayesschen logistischen bzw. linearen multiplen Mehrebenenregression.

Die durchgeführte Mehrebenenregression mit Studierenden als Ebene 1 und Studiengängen als Ebene 2 berücksichtigt dabei die hierarchische Struktur von Studierenden in verschiedenen Studiengängen mit einem Random-Intercept-Modell, d.h. einem über die Studiengänge variierenden Intercept. Eine praktische Einführung zur bayesschen Mehrebenenregression gibt Bürkner (2018), eine Einführung zu Mehrebenenregression in Bildungsforschung gibt Theobald (2018). Wir haben einen bayesschen Ansatz vor allem wegen der Interpretierbarkeit von Kredibilitätsintervallen und Hypothesentests gewählt. Einen einführenden Vergleich klassischer und bayesscher Statistik gibt z. B. Tschirk (2014). Der auf die Ebenenstruktur entfallende Varianzanteil wird über die Intraklassen-Korrelation (ICC) des entsprechenden Null-Modells berechnet und als eine gängige Kenngröße berichtet. Ein RandomSlope-Ansatz für den Effekt der Vorkursteilnahme wird mit dem reinen RandomIntercept-Ansatz verglichen, um zusätzlich einen variierenden Effekt der Vorkursteilnahme nach besuchtem Studiengang zu prüfen.

Es können so wie skizziert verschiedene Modelle verglichen werden: Ein Regressionsmodell mit Random-Intercept erlaubt dabei einen je nach Studiengang variierenden Intercept, d.h. ein bei ansonsten gleichen Merkmalsausprägungen unterschiedliches Niveau der abhängigen Variablen wie z. B. der Abschlussnote. Wir berichten daher Vergleiche der jeweiligen Modelle mit und ohne Random-Intercept, um zu prüfen, ob eine Berücksichtigung der Studiengangsebene zu präferieren ist oder nicht. Die Regressionsgewichte der unabhängigen Variablen sind in einem Random-Intercept-Modell dabei über alle Studiengänge hinweg konstant, d.h. z.B., 
dass die Wirkung des Vorkursbesuches als über alle Studiengänge hinweg identisch modelliert wird. Einen Schritt weiter gehen wir, wenn wir zusätzlich einen Random-Slope-Ansatz für die unabhängige Variable der Vorkursteilnahme prüfen: Hier wird ein über die Studiengänge hinweg variierendes Regressionsgewicht zugelassen, welches zur Prüfung eines entsprechend unterschiedlichen Effekts genutzt werden kann.

Alle nicht-kategorialen Variablen gehen an der Stichprobe standardisiert ein. Effektstärken der logistischen Regressionen werden als Odd Ratios (OR) angegeben. Die Hypothesen werden gegen ein Signifikanzniveau von $5 \%$ getestet, was einer Betrachtung des $95 \%$-Kredibilitätsintervalls (CI) entspricht. Modellvergleiche erfolgen anhand der expected log predictive density (elpd) einer Leave-One-Out-Kreuzvalidierung (LOO-CV, Vehtari et al. 2017), wobei kleinere elpd $\mathrm{loo}_{-}$-Werte das präferierte Modell auszeichnen. Es wird bei Modellvergleichen jeweils die Differenz $\Delta \operatorname{elpd}_{1 \text { oo }}$ der Werte angegeben. Bzgl. der Anpassungsgüte wird das bayessche $R^{2}$ (Gelman et al. 2019) und im Fall logistischer Regression zusätzlich die Area Under the Curve (AUC) berichtet.

Treatment- und Kontrollgruppe (Vorkurs-Teilnehmende und Nicht-Teilnehmende) wurden durch Selbstselektion gebildet. Möglichen Verzerrungen der Schätzung des Treatmenteffekts auf die Teilnehmenden (Average Treatment Effect on Treated, ATT) wird, soweit mit den vorliegenden Variablen möglich, in einem zweiten Schritt durch eine Gewichtung der Kontrollgruppe mittels Entropie-Balancierung begegnet (Hainmueller 2012; Qingyuan und Daniel 2017). Die Balancierung bedeutet hier hinsichtlich der eingehenden Variablen eine Angleichung der Mittelwerte in beiden Gruppen zu erreichen, was u. a. dem Gedanken randomisierter Studiendesigns folgt. Die hierzu bestimmten Gewichte der einzelnen Fälle finden dann Eingang in eine gewichtete Regression.

Es wird so eine Interpretationsmöglichkeit des geschätzten Effekts gefördert. Entropie-Balancierung hat Tieben (2019) in ihrer Untersuchung zu Vorkurseffekten ebenfalls eingesetzt, um auf Verzerrungen aufgrund von Selektion einzugehen. Das alternativ häufig eingesetzte Propensity-Score-Matching (PSM), wie z. B. bei Lesik et al. (2015), steht in der Kritik (King und Nielsen 2019).

Die statistische Auswertung erfolgte in R (Version 3.6.1, R Core Team 2019), primär mit brms (Version 2.12.0, Bürkner 2017, 2018)). Als Priors der bayesschen Regression wurden die in brms gewählten Standardpriors beibehalten. Die EntropieBalancierung wird mit ebal (Version 0.1-6, Hainmueller 2014) via WeightIt (Version 0.9.0, Greifer 2020) vorgenommen.

\section{Ergebnisse}

Die Auswertung wird entlang der Forschungsfragen und im Falle des Studienerfolgs entlang der Operationalisierung von Abschluss und Abschlussnote berichtet. Der Frage nach Interaktionseffekten wird jeweils an den entsprechenden Stellen als Erweiterung des Modells nachgegangen. 
Tab. 2 Teilnehmende und Nicht-Teilnehmende des mathematischen Vorkursangebots. Angegeben sind Mittelwerte mit Standardabweichung bzw. Anzahl im Fall kategorialer Variablen und das standardisierte Odds Ratio (OR) einer jeweils einfachen Regression mit Vorkursteilnahme als abhängiger Variablen.

\begin{tabular}{|c|c|c|c|}
\hline \multirow[b]{2}{*}{ Variable } & \multicolumn{2}{|c|}{ Vorkurs-Teilnahme } & \multirow[b]{2}{*}{ OR mit $95 \%$-CI } \\
\hline & $\begin{array}{l}\mathrm{Ja} \\
(n=1729)\end{array}$ & $\begin{array}{l}\text { Nein } \\
(n=1224)\end{array}$ & \\
\hline$\overline{\text { HZB-Note }}$ & $2,40(0,61)$ & $2,57(0,61)$ & $0,76[0,70 ; 0,82]^{*}$ \\
\hline $\begin{array}{l}\text { Zeit seit } \\
\mathrm{HZB}^{\mathrm{a}}\end{array}$ & $364(447)$ & $370(493)$ & $0,99[0,92 ; 1,06]$ \\
\hline \multicolumn{4}{|l|}{ Geschlecht: } \\
\hline - weiblich & 686 & 456 & \\
\hline - männlich & 1043 & 768 & $0,90[0,78 ; 1,05]$ \\
\hline \multicolumn{4}{|l|}{ Bundesland: } \\
\hline - Nds. & 997 & 594 & \\
\hline $\begin{array}{l}\text { - Nicht } \\
\text { Nds. }\end{array}$ & 732 & 630 & $0,69[0,60 ; 0,80]^{*}$ \\
\hline
\end{tabular}

a in Tagen, ${ }^{*}$ Signifikanzniveau: $5 \%$

\subsection{Vorkursteilnahme}

Eine Übersicht über Teilnehmende und Nicht-Teilnehmende gibt Tab. 2. Insgesamt nahmen 59\% der betrachteten Studienanfängerinnen und -anfänger das mathematische Vorkursangebot wahr.

Das multiple logistische Random-Intercept-Modell der Vorkursteilnahme mit Studiengang als Ebene (Tab. $3 ; R^{2}=0,13 ; 95 \%$-CI $[0,11 ; 0,15]$; AUC $=0,71$ ) ist einem entsprechenden Null-Modell überlegen $\left(\Delta \mathrm{elpd}_{\mathrm{loo}}=-17,70 ; \mathrm{SE}=6,57\right.$; Modellgewichtung: 1,00, 0,00). Die Schätzung der ICC des Null-Modells schließt rechnerisch negative Werte ein $(-1 \%, 95 \%$-CI $[-4 ; 7])$, wobei nur nicht-negative Werte angenommen werden können. Auch bei diesem nicht sicher von null verschiedenen Varianzanteil, der auf die Studiengangsebene entfällt, zeigt ein Modellvergleich von multiplem Modell mit und ohne Random-Intercept für den Studiengang, dass die Ebenenstruktur einbezogen werden sollte $\left(\Delta \operatorname{elpd}_{\mathrm{loo}}=-146,50, \mathrm{SE}=16,67\right.$, Modellgewichtung: 1,00, 0,00).

Das Modell (Tab. 3) zeigt, dass die Teilnahmewahrscheinlichkeit mit besseren HZB-Noten steigt und sie für weibliche Studierende höher ausgeprägt ist, als für

Tab. 3 Bayessche logistische multiple Mehrebenenregression mit Random-Intercept auf Studiengangsebene mit Vorkurs-Teilnahme als abhängiger Variable

\begin{tabular}{lcl}
\hline Variable & $\beta$ mit 95\%-CI & OR $\beta$ mit 95\%-CI \\
\hline Intercept & $0,62[0,21 ; 1,02]$ & $1,86[1,24 ; 2,77]^{*}$ \\
HZB-Note & $-0,18[-0,27 ;-0,08]$ & $0,84[0,76 ; 0,92]^{*}$ \\
Zeit seit HZB & $0,04[-0,04 ; 0,12]$ & $1,04[0,96 ; 1,13]$ \\
Geschlecht: & $-0,29[-0,46 ;-0,12]$ & $0,75[0,63 ; 0,89]^{*}$ \\
männlich & \\
Bundesland: & $-0,37[-0,54 ;-0,21]$ & $0,69[0,58 ; 0,81]^{*}$ \\
Nicht Nds. & \\
Gruppen-Ebene: & \\
Studiengang & & \\
SD(Intercept) & $0,75[0,50 ; 1,14]$ & \\
\hline
\end{tabular}

* Signifikanzniveau: $5 \%$ 
männliche. Studierende aus Niedersachsen, dem Bundesland der betrachteten Universität, nehmen wahrscheinlicher das Vorkursangebot wahr als Studierende von außerhalb des Bundeslands. Für die Zeitdauer seit HZB-Erwerb bis zum Studienbeginn zeigt sich kein signifikanter Effekt auf die Vorkursteilnahme.

Zur Veranschaulichung betrachten wir exemplarisch die Teilnahmewahrscheinlichkeit in Abhängigkeit der (hier nicht standardisierten) HZB-Note. Alle nicht genannten Parameter nehmen den Referenzwert (Bundesland: Niedersachsen) bzw. 0 ein, ebenso geht der Gruppenleveleffekt mit dem Wert 0 ein: Nach dem Modell haben weibliche Studierende mit einer HZB-Note von 1,0 (sehr gut) eine Teilnahmewahrscheinlichkeit von $74 \% 95 \%$-CI $[64 ; 82]$, mit einer HZB-Note von 3,0 (befriedigend) verringert sich ihre Teilnahmewahrscheinlichkeit auf 61\% 95\%-CI [51; 71]. Im Fall von logistischen Regressionen lässt sich dies nicht direkt anhand der

Tab. 4 Studierenden mit und ohne Studienabschluss im gewählten Studiengang an der gewählten Hochschule acht Semester nach Studienbeginn. Angegeben sind Mittelwerte mit Standardabweichung bzw. Anzahl im Fall kategorialer Variablen und das standardisierte Odds Ratio (OR) einer jeweils einfachen Regression mit Studienabschluss als abhängiger Variablen.

Tab. 5 Bayessche logistische multiple Mehrebenenregression mit Random-Intercept auf Studiengangsebene mit Studienabschluss als abhängiger Variable

\begin{tabular}{llll}
\hline & Absolviert & & \\
Variable & Ja & Nein & OR mit 95\%-CI \\
& $(n=1241)$ & $(n=1712)$ & \\
\hline
\end{tabular}

Vorkursteilnahme:

$\begin{array}{lccc}\text { - Nein } & 450 & 774 & \\ \text { - Ja } & 791 & 938 & 1,45[1,25 ; 1,69]^{*} \\ \text { HZB-Note } & 2,29(0,62) & 2,61(0,58) & 0,58[0,54 ; 0,63]^{*} \\ \text { Zeit seit } & 399(451) & 343(477) & 1,13[1,05 ; 1,22]^{*} \\ \text { HZB }^{\text {a }} & & & \end{array}$

Geschlecht:

- weiblich $\quad 540 \quad 602$

- männlich $701 \quad 1110 \quad 0,70[0,61 ; 0,82]^{*}$

Bundesland:

$\begin{array}{llll}- \text { Nds. } & 654 & 937 & \\ - \text { Nicht } & 587 & 775 & 1,08[0,94 ; 1,26] \\ \text { Nds. } & & & \end{array}$

a in Tagen, ${ }^{*}$ Signifikanzniveau: $5 \%$

\begin{tabular}{lll}
\hline Variable & $\beta$ mit 95\%-CI & OR $\beta$ mit 95\%-CI \\
\hline Intercept & $-0,75[-1,16 ;-0,37]$ & $0,47[0,31 ; 2,72]$ \\
Vorkursteilnahme: & $0,36[0,19 ; 0,54]$ & $1,44[1,21 ; 1,71]^{*}$ \\
Ja & & \\
HZB-Note & $-0,77[-0,87 ;-0,67]$ & $0,46[0,42 ; 0,51]^{*}$ \\
Zeit seit HZB & $0,20[0,11 ; 0,28]$ & $1,22[1,12 ; 1,33]^{*}$ \\
Geschlecht: & $-0,21[-0,38 ;-0,04]$ & $0,81[0,68 ; 0,96]^{*}$ \\
männlich & \\
Bundesland: & $-0,12[-0,29 ; 0,04]$ & $0,88[0,75 ; 1,04]$ \\
Nicht Nds. & \\
Gruppen-Ebene: & \\
Studiengang & & \\
SD(Intercept) & $0,69[0,44 ; 1,06]$ & \\
\hline
\end{tabular}

* Signifikanzniveau: $5 \%$ 
Regressionskoeffizienten bzw. der daraus abgeleiteten Odds Ratios, d.h. der Chancenverhältnisse, aus der Tabelle ablesen.

\subsection{Zusammenhang von Vorkursteilnahme und Studienerfolg}

Der Studienerfolg wird auf zwei Weisen operationalisiert, zum einen binär als Absolvierung des gewählten Studiengangs und zum anderen für die Teilmenge der Absolventinnen und Absolventen über die Abschlussnote.

\subsubsection{Zusammenhang von Vorkursteilnahme und Studienabschluss}

In der Stichprobe schlossen $42 \%$ ihren gewählten Studiengang innerhalb von acht Semestern seit Studienbeginn an der gewählten Hochschule ab. Tab. 4 gibt einen Überblick über die beiden Teilgruppen von Studierenden mit und ohne Studienabschluss.

Auch hier wird das multiple logistische Random-Intercept-Modell (Tab. 5; $R^{2}=$ $0,15 ; 95 \%$-CI $[0,13 ; 0,17]$; AUC $=0,72)$ gegenüber einem entsprechenden Null-Modell präferiert $\left(\Delta \mathrm{elpd}_{\mathrm{loo}}=-136,18 ; \mathrm{SE}=16,28\right.$; Modellgewichtung: 1,00, 0,00). Die ICC des Null-Modells zeigt, dass ein Anteil von 6\% 95\%-CI [-1; 19] der Varianz auf die Studiengangsebene entfällt. Ein Modellvergleich von multiplem Modell mit und ohne Random-Intercept für den Studiengang zeigt, dass die Ebenenstruktur einbezogen werden sollte $\left(\Delta \operatorname{elpd}_{\text {loo }}=-92,79 ; \mathrm{SE}=13,11\right.$; Modellgewichtung: $1,00,0,00)$.

Das Modell (Tab. 5) zeigt einen positiven Effekt der Teilnahme an einem mathematischen Vorkurs zu Studienbeginn auf den Studienabschluss acht Semester später im gewählten Studiengang an der gewählten Hochschule. Gegebenenfalls positivverzerrenden Effekten wurde durch die Definition der Stichprobe begegnet (s. o.), die nur Studierende mit aktiver Studienaufnahme umfasst. Daneben zeigt sich für die Kontrollvariablen, dass Studierende mit besseren HZB-Noten und größerer Zeitdauer von HZB-Erwerb bis Studienbeginn wahrscheinlicher ihr gewähltes Studium absolvieren. Weibliche Studierende absolvieren gegenüber männlichen Studierenden wahrscheinlicher den Studiengang. Für das Bundesland wird kein signifikanter Effekt gefunden.

Zur Veranschaulichung betrachten wir den Abschluss in Abhängigkeit der Vorkursteilnahme. Alle nicht genannten Parameter nehmen den Referenzwert (Bundesland: Niedersachsen, Geschlecht: weiblich) bzw. 0 ein, ebenso geht der Gruppenleveleffekt mit dem Wert 0 ein: Vorkursteilnehmende haben nach dem Modell dann eine Abschlusswahrscheinlichkeit von $41 \% 95 \%$-CI [31; 50], Nicht-Teilnehmende haben eine Abschlusswahrscheinlichkeit von 32\% 95\%-CI [24; 41]. Im Fall von logistischen Regressionen lässt sich dies nicht direkt anhand der Regressionskoeffizienten bzw. der daraus abgeleiteten Odds Ratios, d.h. der Chancenverhältnisse, ablesen.

Ein ggf. variierender Effekt des Vorkursbesuchs je nach Studiengang lässt sich modellieren, indem in der Mehrebenenregression zusätzlich zum Random-InterceptAnsatz für den Vorkursbesuch ein Random-Slope eingeführt wird. Das Modell mit zusätzlichem Random-Slope für den Vorkursbesuch zeigt eine dem reinen Ran- 
Tab. 6 Bayessche logistische multiple Mehrebenenregression mit Random-Intercept auf Studiengangsebene und Interaktionsterm mit Studienabschluss als abhängiger Variable

\begin{tabular}{lcl}
\hline Variable & $\beta$ mit 95\%-CI & OR $\beta$ mit 95\%-CI \\
\hline Intercept & $-0,75[-1,18 ;-0,37]$ & $0,47[0,31 ; 0,69]^{*}$ \\
Vorkursteilnahme: & $0,36[0,19 ; 0,54]$ & $1,43[1,20 ; 1,71]^{*}$ \\
Ja & & \\
Zeit seit HZB & $0,08[-0,04 ; 0,20]$ & $1,08[0,96 ; 1,22]$ \\
Vorkursteilnahme & $0,22[0,06 ; 0,39]$ & $1,25[1,06 ; 1,47]^{*}$ \\
$\times$ Zeit seit & \\
HZB & \\
HZB-Note & $-0,78[-0,88 ;-0,68]$ & $0,46[0,41 ; 0,51]^{*}$ \\
Geschlecht: & $-0,22[-0,39 ;-0,05]$ & $0,81[0,68 ; 0,95]^{*}$ \\
männlich & \\
Bundesland: & $-0,12[-0,28 ; 0,05]$ & $0,89[0,75 ; 1,05]$ \\
Nicht Nds. & & \\
Gruppen-Ebene: & & \\
Studiengang & & \\
SD(Intercept) & $0,69[0,45 ; 1,07]$ & \\
\hline
\end{tabular}

* Signifikanzniveau: $5 \%$

dom-Intercept-Ansatz gegenüber nicht zu unterscheidende Güte $\left(\Delta \operatorname{elpd}_{\text {loo }}=-0,33\right.$; $\mathrm{SE}=1,56$; Modellgewichtung: 0,58, 0,42), sodass keine substantiell bessere Modellierung erzielt wird. Damit wird für die vorliegend betrachteten Studiengängen keine substantielle Änderung des Effekts der Vorkursteilnahme zwischen den Studiengängen gefunden.

Die Interaktion von Vorkursteilnahme und Zeitdauer zwischen Erwerb der HZB und Studienaufnahme wird zur Prüfung der entsprechenden Vermutung in das Random-Intercept-Modell aufgenommen (Tab. 6; $R^{2}=0,15 ; 95 \%$-CI $[0,13 ; 0,17]$; AUC $=0,72$ ). Ein Modellvergleich von multiplem Modell mit und ohne Interaktionsterm zeigt, dass die Interaktion einbezogen werden sollte $\left(\Delta \operatorname{elpd}_{\text {loo }}=-2,47\right.$; $\mathrm{SE}=3,32$; Modellgewichtung: 0,92, 0,08). Der Effekt der Vorkursteilnahme auf den Studienabschluss vergrößert sich mit der Zeitdauer zwischen HZB-Erwerb und Studienbeginn.

Die Interaktion von Vorkursteilnahme und HZB-Note wird zur Prüfung in das Random-Intercept-Modell aufgenommen $\left(R^{2}=0,15 ; 95 \%\right.$-CI $[0,13 ; 0,17]$; AUC $=0,72)$. Ein Modellvergleich von multiplem Modell mit und ohne Interaktionsterm zeigt, dass die Interaktion nicht einbezogen werden sollte $\left(\Delta \operatorname{elpd}_{1 \mathrm{oo}}=0,50\right.$; $\mathrm{SE}=0,94$; Modellgewichtung: 0,38, 0,62; $\beta=0,08 ; 95 \%$-CI $[-0,09 ; 0,25]$; $\mathrm{OR}=1,08 ; 95 \%$-CI $[0,91 ; 1,28])$. Eine Interaktion des Effekts der Vorkursteilnahme auf den Studienabschluss mit der HZB-Note wird damit nicht gefunden.

Wie im ersten Teil der Auswertung gesehen, gibt es eine Selektion hinsichtlich der Vorkursteilnahme. Für die Kontrollgruppe wird dementsprechend eine Gewichtung berechnet, die hinsichtlich der Variablen Geschlecht, HZB-Note, Zeitdauer zwischen Erwerb der HZB und Studienbeginn, Bundesland (Niedersachsen) und Studiengang eine Balancierung mit der Treatmentgruppe herstellt, wodurch der effektive Stichprobenumfang der Kontrollgruppe von 1224 auf 673,19 sinkt. Mit dieser Gewichtung werden die multiplen Regressionsanalysen wiederholt. Für die Vorkursteilnahme wird so weiterhin ein positiver Effekt gefunden $(\beta=0,49 ; 95 \%$-CI $[0,35$; 
Tab. 7 Bayessche multiple lineare Mehrebenenregressionen mit Random-Intercept auf Studiengangsebene, mit (Modell 2) und ohne (Modell 1) Interaktionsterm, mit Studienabschlussnote als abhängiger Variable

\begin{tabular}{lll}
\hline Variable & Modell 1 & Modell 2 \\
\hline Intercept & $\beta$ mit 95\%-CI & $\beta$ mit 95\%-CI \\
Vorkursteilnahme: Ja & $-0,01[-0,26 ; 0,24]$ & $-0,01[-0,25 ; 0,23]$ \\
Zeit seit HZB & $-0,14[-0,23 ;-0,04]^{*}$ & $-0,14[-0,23 ;-0,04]^{*}$ \\
Vorkursteilnahme $\times$ Zeit seit HZB & $-0,11[-0,16 ;-0,07]^{*}$ & $-0,06[-0,13 ; 0,01]$ \\
HZB-Note & & $-0,10[-0,19 ;-0,01]^{*}$ \\
Geschlecht: männlich & $0,61[0,56 ; 0,66]^{*}$ & $0,61[0,56 ; 0,66]^{*}$ \\
Bundesland: Nicht Nds. & $0,04[-0,05 ; 0,13]$ & $0,04[-0,05 ; 0,13]$ \\
Gruppen-Ebene: Studiengang & $0,13[0,04 ; 0,22]^{*}$ & $0,12[0,03 ; 0,21]^{*}$ \\
SD(Intercept) & & \\
\hline
\end{tabular}

* Signifikanzniveau: $5 \%$

0,64]; OR=1,64; $95 \%$-CI $[1,42 ; 1,90])$, ebenso wird weiterhin eine positive Interaktion von Vorkursteilnahme und Zeit zwischen HZB-Erwerb und Studienbeginn gefunden $(\beta=0,25 ; 95 \%$-CI $[0,11 ; 0,40]$; OR=1,28; $95 \%$-CI $[1,11 ; 1,48])$, während weiterhin keine Interaktion von Vorkursteilnahme und HZB-Note gefunden wird $(\beta=0,04 ; 95 \%$-CI $[-0,11 ; 0,19]$; OR=1,04; $95 \%$-CI $[0,90 ; 1,21])$.

\subsubsection{Zusammenhang von Vorkursteilnahme und Abschlussnote}

Es wird hier die Teilstichprobe von $n_{a}=1241$ Absolventinnen und Absolventen betrachtet. Diese erzielten im arithmetischen Mittel eine Abschlussnote von 2,21 (0,46). Ein multiples lineares Random-Intercept-Modell (Tab. 7; Modell 1; $R^{2}=0,44 ; 95 \%$-CI $\left.[0,41 ; 0,47]\right)$ wird gegenüber einem entsprechenden Null-Modell präferiert $\left(\Delta \mathrm{elpd}_{\mathrm{loo}}=-213,39\right.$; $\mathrm{SE}=21,06$; Modellgewichtung: 1,00, 0,00). Die ICC des Null-Modells zeigt, dass ein Anteil von $20 \% 95 \%$-CI [10; 29] der Varianz auf die Studiengangsebene entfällt. Ein Modellvergleich von multiplem Modell mit und ohne Random-Intercept auf Studiengangsebene präferiert das Random-Intercept-Modell $\left(\Delta \mathrm{elpd}_{\mathrm{loo}}=-71,04 ; \mathrm{SE}=13,44\right.$; Modellgewichtung: 1,00; 0,00).

Modell 1 (Tab. 7) zeigt einen positiven Effekt von Teilnahme an einem mathematischen Vorkurs zu Studienbeginn auf die Abschlussnote im betrachteten Fach. Daneben zeigt sich für die Kontrollvariablen, dass Studierende mit besseren HZBNoten und größerer Zeitdauer von HZB-Erwerb bis Studienbeginn bessere Abschlussnoten erzielen. Auch das Bundesland der HZB zeigt einen Effekt auf die erzielte Abschlussnote zugunsten von Studierenden mit HZB aus Niedersachsen. Ein signifikanter Effekt des Geschlechts auf die Abschlussnote kann nicht festgestellt werden.

Wie zuvor bei der Modellierung des Studienabschlusses wird bei der Modellierung der Abschlussnote ein Random-Slope-Ansatz für die Vorkursteilnahme gegenüber einem reinen Random-Intercept-Ansatz kontrolliert, um einen ggf. nach Studiengang variierenden Effekt der Vorkursteilnahme auf die Abschlussnote zu prüfen. Das reine Random-Intercept-Modell zeigt hier eine leicht bessere Güte und wird so 
beibehalten $\left(\Delta \mathrm{elpd}_{\mathrm{loo}}=-1,17 ; \mathrm{SE}=0,60\right.$; Modellgewichtung: 0,76, 0,24). Damit wird für die vorliegend betrachteten Studiengänge keine substantielle Änderung des Effekts der Vorkursteilnahme zwischen den Studiengängen gefunden.

Die Interaktion von Vorkursteilnahme und Zeitdauer zwischen Erwerb der HZB und Studienaufnahme wird zur Prüfung der entsprechenden Vermutung in das Random-Intercept-Modell aufgenommen (Tab. 7, Modell 2; $R^{2}=0,44 ; 95 \%$-CI $[0,41$; $0,47]$ ). Es zeigt sich, bei im Modellvergleich von multiplem Modell mit und ohne Interaktionsterm leichter Präferenz für das Modell mit Interaktion $\left(\Delta \mathrm{elpd}_{\mathrm{loo}}=-1,01\right.$; $\mathrm{SE}=2,40$; Modellgewichtung: 0,73, 0,27), eine signifikante Interaktion: Der Effekt der Vorkursteilnahme auf die Abschlussnote vergrößert sich in seinem Betrag mit zunehmender Zeitdauer zwischen HZB-Erwerb und Studienbeginn.

Die Interaktion von Vorkursteilnahme und HZB-Note wird zur Prüfung in das Random-Intercept-Modell aufgenommen $\left(R^{2}=0,44\right.$; $95 \%$-CI $\left.[0,41 ; 0,47]\right)$. Ein Modellvergleich von multiplem Modell mit und ohne Interaktionsterm zeigt, dass die Interaktion nicht einbezogen werden sollte $\left(\Delta \mathrm{elpd}_{1 \mathrm{oo}}=0,58 ; \mathrm{SE}=1,07\right.$; Modellgewichtung: $0,36,0,64 ; \beta=0,04 ; 95 \%$-CI $[-0,04 ; 0,13])$. Eine Interaktion des Effekts der Vorkursteilnahme auf die Studienabschlussnote mit der HZB-Note wird damit nicht gefunden.

Wie zuvor wird für die Kontrollgruppe eine Gewichtung berechnet, die hinsichtlich der Variablen Geschlecht, HZB-Note, Zeitdauer zwischen Erwerb der HZB und Studienbeginn, Bundesland (Niedersachsen) und Studiengang eine Balancierung mit der Treatmentgruppe herstellt. Hierdurch sinkt der effektive Stichprobenumfang der Kontrollgruppe von 450 auf 197,96. Mit dieser Gewichtung werden die multiplen Regressionsanalysen zur Abschlussnote wiederholt. Für die Vorkursteilnahme wird so weiterhin ein positiver Effekt gefunden $(\beta=-0,14 ; 95 \%$-CI $[-0,22$; $-0,06])$. Es wird, anders als zuvor, keine Interaktion von Vorkursteilnahme und Zeit zwischen HZB-Erwerb und Studienbeginn gefunden $(\beta=-0,03 ; 95 \%$-CI $[-0,11$; $0,04])$. Erneut wird keine Interaktion von Vorkursteilnahme und HZB-Note gefunden $(\beta=0,08 ; 95 \%$-CI $[-0,00 ; 0,16])$.

\section{Diskussion}

Motiviert über hohe Studienabbruchquoten wurde der Blick auf Maßnahmen zur Verbesserung des Studienerfolgs gerichtet. Fehlendes Vorwissen bzw. fachliche Überforderung wurde in der Literatur als ein führender Abbruchgrund identifiziert, die Studieneingangsphase als ein relevanter Studienabschnitt benannt. Die Untersuchung der Wirksamkeit mathematischer Vorkurse für Studierende am Übergang in die Universität hinsichtlich ihres langfristigen Studienerfolgs, operationalisiert durch Abschluss nach acht Semestern und Abschlussnote, sowie die Untersuchung möglicher Selektion bzgl. der optionalen Teilnahme, waren Ziel dieser Arbeit.

Es wurde eine Stichprobe von Studierenden der Georg-August-Universität Göttingen betrachtet, für die entsprechende mathematische Vorkurse vor Studienbeginn angeboten wurden.

In den betrachteten Studiengängen mit mathematisch-naturwissenschaftlichen Studieninhalten nahmen rund drei Viertel der Erststudierenden das mathematische 
Vorkursangebot am Übergang in das Universitätsstudium wahr. Verglichen mit in der Literatur berichteten Nutzungsquoten von 44 bis 64 Prozent (Bargel 2015; Tieben 2019) in verschiedenen Stichproben handelt es sich dabei um einen hohen Wert.

Bzgl. der Teilnahme am optionalen Vorkursangebot zeigt sich eine Selektion der Teilnehmenden (Forschungsfrage 1). So stieg die Teilnahmewahrscheinlichkeit mit besseren HZB-Noten an und war für weibliche Studierende höher ausgeprägt als für männliche Studierende. Zusammenhänge der Vorkursteilnahme zum geografischen Einzugsgebiet wurden sichtbar. So nahmen Studierende mit HZB-Erwerb im Bundesland der Hochschule wahrscheinlicher das Vorkursangebot wahr als andere Studierende. Die ebenfalls untersuchte Zeitdauer seit HZB-Erwerb zeigte keinen Zusammenhang zur Vorkursteilnahme.

Die Befunde zur HZB-Note gehen dabei mit denen von Heublein et al. (2010) konform, der ebenfalls eine höhere Teilnahmequote bei besseren HZB-Noten berichtet. Gegenüber den Befunden bei Büchele (2020), dort bezogen auf semesterbegleitende Brückenkurse und bei Tieben (2019), die jeweils keine signifikanten Zusammenhänge von HZB-Note und Teilnahme in ihren Stichproben fanden, legt dies nahe, mögliche Effekte der HZB-Note für die Selektion weiter zu klären. Dieses erscheint, wie bereits Heublein et al. (2010) anmerkt, umso wichtiger, da in der beobachteten Richtung eine ungünstige Steuerung gesehen werden kann, wenn Studierende mit schlechterer HZB-Note Unterstützungsangebote seltener wahrnehmen. Während Büchele (2020) und Tieben (2019) in ihren Stichproben keinen Geschlechtereffekt fanden, motivieren unsere signifikanten Befunde, Hintergründe der selteneren Vorkursteilnahme männlicher Studierender näher zu untersuchen und Effekte ggf. durch Einbezug weiterer Variablen zu erklären. Prüfungen hinsichtlich von geografischen Hürden der Vorkursteilnahme sind in den zitierten Studien nicht vorgenommen worden, sodass die vorliegenden signifikanten Befunde hinsichtlich des Bundeslands der HZB Motivation geben, in künftigen Studien zu Fragen der Selektion als auch zur Kontrolle bei Wirksamkeitsuntersuchungen entsprechende Variablen einzubeziehen. Aus praktischer Perspektive kann hierin ein Problem gesehen werden, wenn die Teilnahme durch geografische Aspekte beschränkt werden sollte. In dieser Hinsicht ist positiv zu bewerten, dass vorliegend ebenso wie bei Büchele (2020) keine Hinweise auf Hürden in Abhängigkeit der Zeit zwischen Schule und Studium gefunden wurden.

Als zentrales Ergebnis wurde entsprechend der Vermutung ein positiver Zusammenhang von Vorkursteilnahme und langfristigem Studienerfolg, sowohl operationalisiert als Studienabschluss acht Semester nach Studienbeginn als auch als Abschlussnote, gefunden (Forschungsfrage 2).

Dieses zeigt, dass mathematische Vorkurse als Maßnahmen zur Steigerung des langfristigen Studienerfolgs in Betracht kommen können. Zugleich zeigen die Auswertungen von Tieben (2019), dass eine positive langfristige Wirkung von Vorkursen nicht sicher ist und einer weiteren Prüfung bedarf, um den Ressourceneinsatz der Institutionen und der Teilnehmenden zu legitimieren. Ein Blick in den US-amerikanischen Raum macht deutlich, dass nicht nur die Möglichkeit von nicht-signifikanten Befunden besteht, sondern sogar auch, in dortiger Ausgestaltung von Förderangeboten, negative Folgen eintreten können, sodass dem Blick auf die langfristige Wirkung 
von Förderangeboten noch mehr Beachtung gewidmet werden sollte, um hier mehr Sicherheit zu gewinnen. Für die Praxis bedeuten die bei uns und bei Tieben (2019) gefundenen Unterschiede, dass die Nicht-Teilnehmenden eine Gruppe mit erhöhtem Risiko bezüglich des langfristigen Studienerfolgs darstellen.

Der Effekt der Vorkursteilnahme auf den Studienabschluss vergrößerte sich entsprechend der Vermutung mit der Zeitdauer zwischen HZB-Erwerb und Studienbeginn, während ein entsprechender Interaktionseffekt hinsichtlich der Abschlussnote sich uneinheitlich zeigte (Forschungsfrage 3a). Der Effekt der Vorkursteilnahme sowohl auf den Studienabschluss als auch auf die Abschlussnote zeigte keine Interaktion mit der HZB-Note (Forschungsfrage 3b).

Ist über die langfristige Wirksamkeit von Vorkursen, wie sie hier betrachtet werden, wenig bekannt, so gilt dies insbesondere für Interaktionseffekte, sowohl mit Merkmalen der Kurse als auch mit Merkmalen der Teilnehmenden. Dass hinsichtlich des Studienabschlusses eine Interaktion der Vorkursteilnahme mit der Zeitdauer zwischen HZB-Erwerb und Studienbeginn der Teilnehmenden bei hier betrachteten Vorkursen, die u. a. der Wiederholung von Schulmathematik dienen, gefunden wurde, stützt dabei konsistent das Gesamtbild unserer Befunde zur Wirksamkeit. Für die Praxis deutet der Befund darauf hin, dass Vorkurse hinsichtlich des Studienabschlusses somit nicht nur, aber insbesondere für die Teilgruppe an Studierenden mit einer größeren Lücke zwischen Schulzeit und Studium eine hilfreiche Maßnahme darstellen können.

\section{Limitationen und Ausblick}

Außerhalb der Reichweite des Studiendesigns lag die empirische Untersuchung, warum und wie die Vorkurse ihre Wirkung erzielen können. Dabei erscheint es neben den Prozessen während der Vorkurse und über kurz- und mittelfristige Betrachtungen hinaus interessant und relevant, empirisch der Frage nachzugehen, wie die hier im Fokus stehende langfristige Wirkung von Vorkursen vermittelt wird. Lassen sich beispielsweise Ketten identifizieren, die von Vorkursbesuch über kurzund mittelfristigen hin zu langfristigen Unterschieden führen? Die positiven statistischen Befunde zu Vorkursbesuch und langfristigem Studienerfolg sollen hier als Motivation für tiefergehende Forschungsansätze aufgefasst werden.

Aufgrund der geringen Anzahl und Überschneidung mit gewählten Studiengängen der verschiedenen Vorkursangebote konnte vorliegend nicht näher auf die für ihre Wirkung auf den Studienerfolg ggf. entscheidenden Oberflächen- und Tiefenmerkmale eingegangen werden. Hier bieten sich Möglichkeiten insbesondere auch für experimentelle Designs. Es wurde lediglich die Vorkursteilnahme in binärer Ausprägung betrachtet, wie z. B. bei Tieben (2019).

Die Studie wurde über verschiedene Studiengänge und Vorkurse, aber an nur einer Universität durchgeführt; für eine Verallgemeinerung der gefundenen Effekte bedarf es daher weiterer Studien an anderen Standorten. Die Forschungsfragen wurden in Bezug auf Studienanfängerinnen und -anfänger am Übergang in das Hochschulsystem gestellt, die Stichprobe wurde entsprechend selektiert. Die Wirkung von Vorkursen beim Studiengangswechsel bedarf einer eigenen Untersuchung. Für die 
Bearbeitung der Forschungsfrage zur Selektion hinsichtlich der Teilnahme an Vorkursen stand nur eine begrenzte Auswahl an Variablen zur Verfügung, zudem ist eine kausale Interpretation von gefundenen Unterschieden aufgrund des vorliegenden Designs nicht möglich. Qualitative Interviews und quantitative Fragebogenstudien sind an dieser Stelle geeignet, den Entscheidungsprozess und insbesondere Gründe und Motive für Teilnahme bzw. Nicht-Teilnahme zu erforschen.

Als Kriterien des langfristigen Studienerfolgs wurden der Studienabschluss innerhalb von acht Semestern nach Studienbeginn im gewählten Studiengang an der gewählten Hochschule und die Abschlussnote betrachtet. Hier sind weitere Operationalisierungen denkbar, wie z.B. erfolgreicher Studienabschluss unabhängig von Studiengang, Hochschule und zeitlichem Rahmen, erworbene Credits oder subjektive Studienzufriedenheit, die ggf. zu anderen Ergebnisse führen. Die HZB-Note wurde als über verschiedene Gruppen vergleichbare Variable in die Modelle aufgenommen. Während dieses üblich und verbreitet ist, möchten wir vermerken, dass es sich nicht um eine einheitliche Messung handelt, sodass Unterschiede auf Ebene u. a. von Arten der HZB, Jahrgängen, (Bundes-)Ländern, Schulen, Lehrkräften und Komposition nicht berücksichtigt werden. Die Aufnahme ergänzender Kompetenzmessungen kann hier hilfreich sein. Die Variable des Bundeslands der HZB, binär als Niedersachsen und Nicht-Niedersachsen codiert, wurde als ein Aggregat u.a. von Einzugsgebieten und bildungspolitischen Rahmenbedingungen aufgenommen; die Interpretationsmöglichkeit der Variable ist damit eingeschränkt. Mit ausdifferenzierten Variablen kann mit bundesweiten Studien eine höhere Auflösung und Interpretierbarkeit erzielt werden. Für die Zeit zwischen Erwerb der HZB und erstmaligem Studienbeginn musste unberücksichtigt bleiben, wodurch die ggf. frühere oder spätere Studienaufnahme bedingt wurde und welche Aktivitäten in der Zwischenzeit ausgeübt wurden. Denkbar ist, dass hierin relevante Merkmale für eine Moderation der Vorkurswirksamkeit liegen.

Für eine Förderung der Interpretationsmöglichkeit wurden Effekte um eine Selektion der Teilnehmenden, soweit mit den vorliegenden Variablen möglich, korrigiert. Es handelt sich jedoch nicht um ein experimentelles Design, auch blieb die Möglichkeit zur Kontrolle durch die zur Verfügung stehenden Variablen begrenzt, sodass ein Nachweis kausaler Zusammenhänge nicht geführt wird, was bei der Interpretation stets zu berücksichtigen ist. Ein experimentelles Design mit randomisierten Treatment- und Kontrollgruppen kann hier Evidenz geben. Da u.a. aus praktischen und ethischen Gründen die Durchführbarkeit solcher experimentellen Designs eingeschränkt ist, kann zur Nutzung der Gelegenheit natürlicher Experimente aufgerufen werden. Als Einschränkung bei der Interpretation ist auch zu bedenken, dass mit der Teilnahme an mathematischen Vorkursen die Nutzung weiterer Maßnahmen und Angebote verbunden sein kann, sei es fachlich, in der Beratung oder sozial.

Ein möglicher Teilnahmeschwund während der Kurse, Haase (2014) berichtet beispielsweise von 30\%, ähnlich auch Kürten (2020), blieb unbeachtet. Fischer (2014) empfiehlt, Abbrechende der Kurse näher zu untersuchen; dieser Empfehlung schließen wir uns an. Für nähere Betrachtungen bieten sich hier AngebotsNutzungs-Modelle an. Einen entsprechenden Analyserahmen für Wirkungsweisen von Angeboten am Studienbeginn zeigen Bosse und Mergner (2019). 
Danksagung Wir bedanken uns bei den am Forschungsprojekt GLA teilnehmenden Studierenden als auch bei Dozentinnen und Dozenten, Mitarbeiterinnen und Mitarbeitern wie auch studentischen Hilfskräften für ihre Beiträge zur Datenaufnahme. Bei den anonymen Gutachtenden bedanken wir uns für ihre wertvolle Beratung.

Authors' contributions Zur Konzeption der Studie und zur Erhebung bzw. Datenbeschaffung haben alle Autorinnen und Autoren beigetragen. Die Auswertung hat A. G. durchgeführt. Eine erste Version des Manuskripts hat A. G. geschrieben. Alle Autorinnen und Autoren haben das Manuskript kommentiert und die eingereichte Version bestätigt.

Funding Open Access funding enabled and organized by Projekt DEAL.

Open Access Dieser Artikel wird unter der Creative Commons Namensnennung 4.0 International Lizenz veröffentlicht, welche die Nutzung, Vervielfältigung, Bearbeitung, Verbreitung und Wiedergabe in jeglichem Medium und Format erlaubt, sofern Sie den/die ursprünglichen Autor(en) und die Quelle ordnungsgemäß nennen, einen Link zur Creative Commons Lizenz beifügen und angeben, ob Änderungen vorgenommen wurden.

Die in diesem Artikel enthaltenen Bilder und sonstiges Drittmaterial unterliegen ebenfalls der genannten Creative Commons Lizenz, sofern sich aus der Abbildungslegende nichts anderes ergibt. Sofern das betreffende Material nicht unter der genannten Creative Commons Lizenz steht und die betreffende Handlung nicht nach gesetzlichen Vorschriften erlaubt ist, ist für die oben aufgeführten Weiterverwendungen des Materials die Einwilligung des jeweiligen Rechteinhabers einzuholen.

Weitere Details zur Lizenz entnehmen Sie bitte der Lizenzinformation auf http://creativecommons.org/ licenses/by/4.0/deed.de.

\section{Conflict of interest}

S.H. hat als wissenschaftlicher Leiter des Bereichs Vorkurse und Propädeutika im Handlungsfeld Studieneingangsphase im Qualitätspakt-Lehre Projekt Campus Q Plus der untersuchten Universität und von Teilangeboten dieses Programms als Dozent u.a. in den betrachteten Kohorten mitgewirkt.

\section{Literatur}

Abel, H., \& Weber, B. (2014). 28 Jahre Esslinger Modell-Studienanfänger und Mathematik. In I. Bausch, R. Biehler, R. Bruder, P. R. Fischer, R. Hochmuth, W. Koepf, S. Schreiber \& T. Wassong (Hrsg.), Mathematische Vor- und Brückenkurse (S. 9-19). Wiesbaden: Springer Spektrum. https://doi.org/10. 1007/978-3-658-03065-0_2.

Albrecht, A., \& Nordmeier, V. (2011). Ursachen des Studienabbruchs in Physik. Eine explorative Studie. Die Hochschule: Journal für Wissenschaft und Bildung, 20(2), 131-145.

Bargel, T. (2015). Studieneingangsphase und heterogene Studentenschaft - neue Angebote und ihr Nutzen: Befunde des 12. Studierendensurveys an Universitäten und Fachhochschulen. Technischer Bericht 83. http://nbn-resolving.de/urn:nbn:de:bsz:352-0-311709. Zugegriffen: 26. Febr. 2021.

Bausch, I., Biehler, R., Bruder, R., Fischer, P. R., Hochmuth, R., Koepf, W., Schreiber, S., \& Wassong, T. (Hrsg.). (2014). Mathematische Vor- und Brückenkurse. Wiesbaden: Springer Spektrum. https://doi. org/10.1007/978-3-658-03065-0.

Binder, T., Sandmann, A., Sures, B., Friege, G., Theyssen, H., \& Schmiemann, P. (2019). Assessing prior knowledge types as predictors of academic achievement in the introductory phase of biology and physics study programmes using logistic regression. International Journal of STEM Education, 6(1), 33. https://doi.org/10.1186/s40594-019-0189-9.

Blömeke, S. (2016). Der Übergang von der Schule in die Hochschule: Empirische Erkenntnisse zu mathematikbezogenen Studiengängen. In A. Hoppenbrock, R. Biehler, R. Hochmuth \& H.-G. Rück (Hrsg.), Lehren und Lernen von Mathematik in der Studieneingangsphase (S. 3-13). Wiesbaden: Springer Spektrum. https://doi.org/10.1007/978-3-658-10261-6_1. 
Blüthmann, I., Lepa, S., \& Thiel, F. (2012). Überfordert, Enttäuscht, Verwählt oder Strategisch? Eine Typologie vorzeitig exmatrikulierter Bachelorstudierender. Zeitschrift für Pädagogik, 58(1), 89-108.

BMBF (2019). Richtlinie zur Förderung von Forschung über 'Studienerfolg und Studienabbruch II'. Bundesanzeiger vom 20.12.2019

Boatman, A., \& Long, B.T. (2018). Does remediation work for all students? How the effects of postsecondary remedial and developmental courses vary by level of academic preparation. Educational Evaluation and Policy Analysis, 40(1), 29-58. https://doi.org/10.3102/0162373717715708.

Bosse, E., \& Mergner, J. (2019). „Und das hat RICHTIG geholfen.“- Wirkungsweisen von Angeboten für den Studieneinstieg. ZeHf-Zeitschrift für empirische Hochschulforschung, 3(2), 105-122. https://doi. org/10.3224/zehf.v3i2.02.

Bosse, E., Mergner, J., Wallis, M., Jänsch, V.K., \& Kunow, L. (2019). Gelingendes Studieren in der Studieneingangsphase. Ergebnisse und Anregungen für die Praxis aus der Begleitforschung zum Qualitätspakt Lehre im Projekt StuFHe. Hamburg: Universitätsdruckerei. https://doi.org/10.25592/ StuFHe2019.

Büchele, S. (2020). Bridging the gap-How effective are remedial math courses in Germany? Studies in Educational Evaluation. https://doi.org/10.1016/j.stueduc.2019.100832.

Burkholder, E.W., Murillo-Gonzalez, G., \& Wieman, C. (2021). Importance of math prerequisites for performance in introductory physics. Physical Review Physics Education Research, 17(1), 10108. https://doi.org/10.1103/PhysRevPhysEducRes.17.010108.

Bürkner, P.-C. (2017). brms: an R package for Bayesian multilevel models using Stan. Journal of Statistical Software, 80(1), 1-28. https://doi.org/10.18637/jss.v080.i01.

Bürkner, P.-C. (2018). Advanced Bayesian multilevel modeling with the R package brms. The R Journal, 10(1), 395-411. https://doi.org/10.32614/RJ-2018-017.

Buschhüter, D., Spoden, C., \& Borowski, A. (2016). Mathematische Kenntnisse und Fähigkeiten von Physikstudierenden zu Studienbeginn. Zeitschrift für Didaktik der Naturwissenschaften, 22(1), 61-75. https://doi.org/10.1007/s40573-016-0041-4.

CHE (2021). Indikator Abschlüsse in angemessener Zeit. https://methodik.che-ranking.de/indikatoren/ abschluesse-in-angemessener-zeit/. Zugegriffen: 26. Febr. 2021.

cosh (2014). Mindestanforderungskatalog Mathematik (Version 2.0) der Hochschulen Baden-Württembergs für ein Studium von WiMINT-Fächern. https://lehrerfortbildung-bw.de/u_matnatech/ mathematik/bs/bk/cosh/katalog/makv20b_ohne_leerseiten.pdf. Zugegriffen: 26. Febr. 2021.

Cramer, E., \& Nešlehová, J. (2018). Vorkurs Mathematik. Berlin, Heidelberg, New York: Springer. https:// doi.org/10.1007/978-3-662-57494-2.

De Paola, M., \& Scoppa, V. (2014). The effectiveness of remedial courses in Italy: a fuzzy regression discontinuity design. Journal of Population Economics, 27(2), 365-386. https://doi.org/10.1007/s00148013-0466-8.

DMV, GDM, \& MNU (2019). Mathematik: 19 Maßnahmen für einen konstruktiven Übergang Schule Hochschule. https://www.mathematik.de/images/Presse/Presseinformationen/Massnahmenkatalog_ DMV_GDM_MNU.pdf. Zugegriffen: 26. Feb. 2021.

Fischer, P. R. (2014). Mathematische Vorkurse im Blended-Learning-Format: Konstruktion, Implementation und wissenschaftliche Evaluation. Wiesbaden: Springer Spektrum. https://doi.org/10.1007/978-3658-05813-5.

Fleischer, J., Leutner, D., Brand, M., Fischer, H., Lang, M., Schmiemann, P., \& Sumfleth, E. (2019). Vorhersage des Studienabbruchs in naturwissenschaftlich-technischen Studiengängen. Zeitschrift für Erziehungswissenschaft, 22(5), 1077-1097. https://doi.org/10.1007/s11618-019-00909-w.

Freyer, K., Epple, M., Brand, M., Schiebener, J., \& Sumfleth, E. (2014). Studienerfolgsprognose bei Erstsemesterstudierenden in Chemie. Zeitschrift für Didaktik der Naturwissenschaften, 20(1), 129-142. https://doi.org/10.1007/s40573-014-0015-3.

Gelman, A., Goodrich, B., Gabry, J., \& Vehtari, A. (2019). R-squared for Bayesian regression models. The American Statistician, 73(3), 307-309. https://doi.org/10.1080/00031305.2018.1549100.

Georg-August-Universität Göttingen (Hrsg.). (1969). Vorlesungsverzeichnis Wintersemester 1969/70. Göttingen: Vandenhoeck \& Ruprecht. http://resolver.sub.uni-goettingen.de/purl?PPN830250301_1969_ 1970_WS

Greefrath, G., Hoever, G., Kürten, R., \& Neugebauer, C. (2015). Vorkurse und Mathematiktests zu Studienbeginn-Möglichkeiten und Grenzen. In J. Roth, T. Bauer, H. Koch \& S. Prediger (Hrsg.), Übergänge konstruktiv gestalten (S. 19-32). Wiesbaden: Springer Spektrum. https://doi.org/10.1007/978-3-65806727-4_2.

Greefrath, G., Koepf, W., \& Neugebauer, C. (2017). Is there a link between preparatory course attendance and academic success? A case study of degree programmes in electrical engineering and computer 
science. International Journal of Research in Undergraduate Mathematics Education, 3(1), 143-167. https://doi.org/10.1007/s40753-016-0047-9.

Greifer, N. (2020). WeightIt: Weighting for Covariate Balance in Observational Studies. R package version 0.9.0. https://CRAN.R-project.org/package=WeightIt. Zugegriffen: 12. Apr. 2020.

Haase, D. (2014). Studieren im MINT-Kolleg Baden-Württemberg. In I. Bausch, R. Biehler, R. Bruder, P.R. Fischer, R. Hochmuth, W. Koepf, S. Schreiber \& T. Wassong (Hrsg.), Mathematische Vorund Brückenkurse (S. 123-136). Wiesbaden: Springer Spektrum. https://doi.org/10.1007/978-3-65803065-0_9.

Hainmueller, J. (2012). Entropy balancing for causal effects: a multivariate reweighting method to produce balanced samples in observational studies. Political Analysis, 20(1), 25-46.

Hainmueller, J. (2014). ebal: Entropy reweighting to create balanced samples. R package version 0.1-6. https://CRAN.R-project.org/package=ebal. Zugegriffen: 6. Nov. 2020.

Halverscheid, S., \& Pustelnik, K. (2013). Studying math at the university: is dropout predictable? In A. Lindmeier \& A. Heinze (Hrsg.), Proceedings of the 37th Conference of the International Group for Psychology of Mathematics Education (Bd. 2, S. 417-424).

Halverscheid, S., Pustelnik, K., Schneider, S., \& Taake, A. (2014). Ein diagnostischer Ansatz zur Ermittlung von Wissenslücken zu Beginn mathematischer Vorkurse. In I. Bausch, R. Biehler, R. Bruder, P. R. Fischer, R. Hochmuth, W. Koepf, S. Schreiber \& T. Wassong (Hrsg.), Mathematische Vorund Brückenkurse (S. 295-308). Wiesbaden: Springer Spektrum. https://doi.org/10.1007/978-3-65803065-0_20.

Hefft, K. (2018). Mathematischer Vorkurs zum Studium der Physik. Berlin: Springer Spektrum. https://doi. org/10.1007/978-3-662-53831-9.

Heublein, U., Ebert, J., Hutzsch, C., Isleib, S., König, R., Richter, J., \& Woisch, A. (2017). Zwischen Studienerwartungen und Studienwirklichkeit. Ursachen des Studienabbruchs, beruflicher Verbleib der Studienabbrecherinnen und Studienabbrecher und Entwicklung der Studienabbruchquote an deutschen Hochschulen. Hannover: DZHW. Nr. 112017 in Forum Hochschule

Heublein, U., Hutzsch, C., Schreiber, J., Sommer, D., \& Besuch, G. (2010). Ursachen des Studienabbruchs in Bachelor- und in herkömmlichen Studiengängen. Ergebnisse einer bundesweiten Befragung von Exmatrikulierten des Studienjahres. Hannover: HIS. Nr. 212010 in Forum Hochschule

Hoppenbrock, A., Biehler, R., Hochmuth, R., \& Rück, H.-G. (Hrsg.). (2016). Lehren und Lernen von Mathematik in der Studieneingangsphase. Wiesbaden: Springer Spektrum. https://doi.org/10.1007/ 978-3-658-10261-6.

Kallweit, M., Dehling, H., \& Härterich, J. (2018). Einsatz von mathematischen Vorkursen. Vortrag im Rahmen der 43. Plenarversammlung der Konferenz der Mathematischen Fachbereiche. http://kmathf. math.uni-bielefeld.de/plenum/Einsatz_von_mathematischen_Vorkursen.pdf. Zugegriffen: 26. Febr. 2021.

Kehm, B. M., Larsen, M.R., \& Sommersel, H.B. (2019). Student dropout from universities in Europe: a review of empirical literature. Hungarian Educational Research Journal, 9(2), 147-164. https://doi. org/10.1556/063.9.2019.1.18.

Key, O., \& Hill, L. (2018). Modellansätze ausgewählter Hochschulen zur Neugestaltung der Studieneingangsphase. Berlin: Hochschulrektorenkonferenz. https://www.hrk-nexus.de/fileadmin/redaktion/ hrk-nexus/07-Downloads/07-02-Publikationen/CHE_07032018_final.pdf. Zugegriffen: 26. Febr. 2021.

KFP (2012). Empfehlung der Konferenz der Fachbereiche Physik zum Umgang mit den Mathematikkenntnissen von Studienanfängern der Physik. https://www.kfp-physik.de/dokument/KFP-EmpfehlungMathematikkenntnisse.pdf. Zugegriffen: 26. Febr. 2021.

King, G., \& Nielsen, R. (2019). Why propensity scores should not be used for matching. Political Analysis, 27(4), 435-454. https://doi.org/10.1017/pan.2019.11.

Klein, P., Kuhn, J., \& Müller, A. (2018). Förderung von Repräsentationskompetenz und Experimentbezug in den vorlesungsbegleitenden Übungen zur Experimentalphysik. Zeitschrift für Didaktik der Naturwissenschaften, 24(1), 17-34. https://doi.org/10.1007/s40573-018-0070-2.

Krause, F., \& Reiners-Logothetidou, A. (1981). Kenntnisse und Fähigkeiten naturwissenschaftlich orientierter Studienanfänger in Physik und Mathematik: Die Ergebnisse des bundesweiten Studieneingangstests Physik 1978. Universität Bonn.

Kürten, R. (2020). Mathematische Unterstützungsangebote für Erstsemesterstudierende. Wiesbaden: Springer Spektrum. https://doi.org/10.1007/978-3-658-30225-2.

Larsen, M.S., Kornbeck, K.P., Kristensen, R.M., Larsen, M.R., \& Sommersel, H. B. (2013). Dropout phenomena at universities: what is dropout? Why does dropout occur? What can be done by the universities to prevent or reduce it? A systematic review. https://edu.au.dk/fileadmin/edu/Udgivelser/ 
Clearinghouse/Review/Evidence_on_dropout_from_universities_technical_report_May_2013.pdf. Zugegriffen: 26. Febr. 2021.

Lesik, S. A., Santoro, K. G., \& DePeau, E. A. (2015). Evaluating the effectiveness of a mathematics bridge program using propensity scores. Journal of Applied Research in Higher Education, 7, 331-345. https://doi.org/10.1108/JARHE-01-2014-0010.

Moser-Fendel, J., \& Wessel, L. (2019). Relevante Fakten zum Übergang Schule-Hochschule. Mitteilungen der Gesellschaft für Didaktik der Mathematik, 45(107), 8-21.

Moss, B. G., Yeaton, W.H., \& LIoyd, J.E. (2014). Evaluating the effectiveness of developmental mathematics by embedding a randomized experiment within a regression discontinuity design. Educational Evaluation and Policy Analysis, 36(2), 170-185. https://doi.org/10.3102/0162373713504988.

Müller, J., Stender, A., Fleischer, J., Borowski, A., Dammann, E., Lang, M., \& Fischer, H.E. (2018). Mathematisches Wissen von Studienanfängern und Studienerfolg. Zeitschrift für Didaktik der Naturwissenschaften, 24(1), 183-199. https://doi.org/10.1007/s40573-018-0082-y.

MWK (2019). MINT in Niedersachsen - Mathematik für einen erfolgreichen Studienstart. Basispapier Mathematik. Ergebnis des Institutionalisierten Gesprächskreises Mathematik Schule-Hochschule IGeMa. Hannover: MWK.

Neugebauer, M., Heublein, U., \& Daniel, A. (2019). Studienabbruch in Deutschland: Ausmaß, Ursachen, Folgen, Präventionsmöglichkeiten. Zeitschrift für Erziehungswissenschaft, 22(5), 1025-1046. https:// doi.org/10.1007/s11618-019-00904-1.

Neumann, I., Pigge, C., \& Heinze, A. (2017). Welche mathematischen Lernvoraussetzungen erwarten Hochschullehrende für ein MINT-Studium? Kiel: IPN-Leibniz-Institut für die Pädagogik der Naturwissenschaften und Mathematik.

Purkert, W. (2014). Brückenkurs Mathematik für Wirtschaftswissenschaftler. Wiesbaden: Gabler. https:// doi.org/10.1007/978-3-8348-2325-0.

Qingyuan, Z., \& Daniel, P. (2017). Entropy balancing is doubly robust. Journal of Causal Inference, 5(1). https://doi.org/10.1515/jci-2016-0010.

R Core Team (2019). R: a language and environment for statistical computing. R Foundation for Statistical Computing.

Rach, S. (2014). Charakteristika von Lehr-Lern-Prozessen im Mathematikstudium: Bedingungsfaktoren für den Studienerfolg im ersten Semester. Münster: Waxmann.

Rach, S., \& Heinze, A. (2017). The transition from school to university in mathematics: Which influence do school-related variables have? International Journal of Science and Mathematics Education, 15(7), 1343-1363. https://doi.org/10.1007/s10763-016-9744-8.

Rach, S., Heinze, A., \& Ufer, S. (2014). Welche mathematischen Anforderungen erwarten Studierende im ersten Semester des Mathematikstudiums? Journal für Mathematik-Didaktik, 35(2), 205-228. https:// doi.org/10.1007/s13138-014-0064-7.

Rach, S., \& Ufer, S. (2020). Which prior mathematical knowledge is necessary for study success in the university study entrance phase? Results on a new model of knowledge levels based on a reanalysis of data from existing studies. International Journal of Research in Undergraduate Mathematics Education, 6(3), 375-403. https://doi.org/10.1007/s40753-020-00112-x.

Reichersdorfer, E., Ufer, S., Lindmeier, A., \& Reiss, K. (2014). Der Übergang von der Schule zur Universität: Theoretische Fundierung und praktische Umsetzung einer Unterstützungsmaßnahme am Beginn des Mathematikstudiums. In I. Bausch, R. Biehler, R. Bruder, P. R. Fischer, R. Hochmuth, W. Koepf, S. Schreiber \& T. Wassong (Hrsg.), Mathematische Vor- und Brückenkurse (S. 37-53). Wiesbaden: Springer Spektrum. https://doi.org/10.1007/978-3-658-03065-0_4.

Rolf, B., Lankeit, E., Neuhaus, S., Hochmuth, R., Kuklinski, C., Leis, E., Liebendörfer, M., Schaper, N., \& Schürmann, M. (2018). Different goals for pre-university mathematical bridging courses-Comparative evaluations, instruments and selected results. In V. Durand-Guerrier, R. Hochmuth, S. Goodchild \& N.M. Hogstad (Hrsg.), Proceedings of the Second Conference of the International Network for Didactic Research in University Mathematics (INDRUM 2018, 5-7 April 2018, Kristiansand, Norway) (S. 467-476). University of Agder and INDRUM.

Roth, J., Bauer, T., Koch, H., \& Prediger, S. (Hrsg.). (2015). Übergänge konstruktiv gestalten. Wiesbaden: Springer Spektrum. https://doi.org/10.1007/978-3-658-06727-4.

Schaeper, H. (2020). The first year in higher education: the role of individual factors and the learning environment for academic integration. Higher Education, 79(1), 95-110. https://doi.org/10.1007/s 10734019-00398-0.

Schnoor, B. (2012). Vorkurs zur Vorlesung Mathematik für Wirtschaftswissenschaftler (Stand 19.07.2012). Georg-August-Universität Göttingen. https://www.uni-goettingen.de/de/document/download/a9d5e9 
4b54cce7a5f4ea40df310622b3.pdf/Inhalt\%20Mathevorkurs_Stand_19.07.pdf. Zugegriffen: 30. Aug. 2021.

Theobald, E. (2018). Students are rarely independent: when, why, and how to use random effects in discipline-based education research. CBE_Life Sciences Education, 17(3), rm2. https://doi.org/10.1187/ cbe.17-12-0280.

Tieben, N. (2019). Brückenkursteilnahme und Studienabbruch in Ingenieurwissenschaftlichen Studiengängen. Zeitschrift für Erziehungswissenschaft, 22(5), 1175-1202. https://doi.org/10.1007/s11618-01900906-z.

Trapmann, S., Hell, B., Weigand, S., \& Schuler, H. (2007). Die Validität von Schulnoten zur Vorhersage des Studienerfolgs-eine Metaanalyse. Zeitschrift für pädagogische Psychologie, 21(1), 11-27. https:// doi.org/10.1024/1010-0652.21.1.11.

Tschirk, W. (2014). Statistik: Klassisch oder Bayes. Berlin: Springer Spektrum. https://doi.org/10.1007/ 978-3-642-54385-2.

Valentine, J.C., Konstantopoulos, S., \& Goldrick-Rab, S. (2017). What happens to students placed into developmental education? A meta-analysis of regression discontinuity studies. Review of Educational Research, 87(4), 806-833. https://doi.org/10.3102/0034654317709237.

Vehtari, A., Gelman, A., \& Gabry, J. (2017). Practical Bayesian model evaluation using leave-one-out cross-validation and WAIC. Statistics and computing, 27(5), 1413-1432. https://doi.org/10.1007/ s11222-016-9696-4.

Voßkamp, R., \& Laging, A. (2014). Teilnahmeentscheidungen und Erfolg. In I. Bausch, R. Biehler, R. Bruder, P. R. Fischer, R. Hochmuth, W. Koepf, S. Schreiber \& T. Wassong (Hrsg.), Mathematische Vor- und Brückenkurse (S. 67-83). Wiesbaden: Springer Spektrum. https://doi.org/10.1007/978-3658-03065-0_6.

Wissenschaftsrat (2018). Hochschulbildung im Anschluss an den Hochschulpakt 2020. Positionspapier (Drs. 7013-18). https://www.wissenschaftsrat.de/download/archiv/7013-18.html. Zugegriffen: 26. Febr. 2021. 